Draft VERSiOn June 13, 2018

Preprint typeset using $\mathrm{LAT}_{\mathrm{E}} \mathrm{X}$ style emulateapj v. 12/16/11

\title{
SHOCKED INTERSTELLAR CLOUDS AND DUST GRAIN DESTRUCTION IN THE LMC SUPERNOVA REMNANT N132D
}

\author{
Michael A. Dopita ${ }^{1,2}$, Frédéric P.A. Vogt ${ }^{3,4}$, Ralph S. Sutherland ${ }^{1,2}$, \\ Ivo R. Seitenzahl ${ }^{5,6}$, Ashley J. Ruiter ${ }^{5,6}$ \& Parviz Ghavamian ${ }^{7}$ \\ Draft version June 13, 2018
}

\begin{abstract}
From integral field data we extract the optical spectra of 20 shocked clouds in the supernova remnant N132D in the Large Magellanic Cloud (LMC). Using self-consistent shock modelling, we derive the shock velocity, pre-shock cloud density and shock ram pressure in these clouds. We show that the [Fe $\mathrm{X}]$ and [Fe XIV] emission arises in faster, partially radiative shocks moving through the lower density gas near the periphery of the clouds. In these shocks dust has been effectively destroyed, while in the slower cloud shocks the dust destruction is incomplete until the recombination zone of the shock has been reached. These dense interstellar clouds provide a sampling of the general interstellar medium (ISM) of the LMC. Our shock analysis allows us to make a new determination of the ISM chemical composition in $\mathrm{N}, \mathrm{O}, \mathrm{Ne}, \mathrm{S}, \mathrm{Cl}$ and $\mathrm{Ar}$, and to obtain accurate estimates of the fraction of refractory grains destroyed. From the derived cloud shock parameters, we estimate cloud masses and show that the clouds previously existed as typical self-gravitating Bonnor-Ebert spheres into which converging cloud shocks are now being driven.

Subject headings: physical data and processes: radiation transfer, shock waves, ISM: supernova remnants, abundances, galaxies: Magellanic Clouds
\end{abstract}

\section{INTRODUCTION}

The supernova remnant (SNR) N132D in the Large Magellanic Cloud (LMC) is the brightest X-ray emitting SNR in this galaxy (Hwang et al. 1993, Favata et al. 1997, Hughes et al. 1998; Borkowski et al. 2007; Xiao \& Chen 2008). Located within the stellar bar of the LMC, N132D was first identified as a SNR by Westerlund \& Mathewson (1966) on the basis of the association of a non-thermal radio source with a [S II]- bright optical structure. Danziger \& Dennefeld (1976) discovered high-velocity $[\mathrm{O}$ III $]$ and $[\mathrm{Ne}$ III $]$ material ejected in the supernova explosion, which established N132D as a relatively young SNR in which the reverse shock has not yet reached the centre of the remnant, marking the formal transition to the Sedov phase of evolution. This would imply that the swept-up interstellar medium (ISM) is less than a few times the mass ejected in the supernova event, a result which is in apparent contradiction to the swept-up mass derived from X-ray observations (Hughes et al. 1998). From the dynamics of the fast-moving $O$ and Ne material, Danziger \& Dennefeld (1976) derived a maximum age of $3440 \mathrm{yr}$ for the SNR, and a probable age of $\sim 1350$ yr. Subsequent analyses gave similar ages; 2350 yr (Lasker 1980), and 2500 yr (Vogt \& Dopita 2011).

\footnotetext{
${ }^{1}$ Research School of Astronomy and Astrophysics, Australian National University, Cotter Road, Weston Creek, ACT 2611, Australia.

${ }^{2}$ ARC Centre of Excellence for All Sky Astrophysics in 3 Dimensions (ASTRO 3D).

${ }^{3}$ European Southern Observatory, Av. Alonso de Cordova 3107, 7630355 Vitacura, Santiago, Chile.

${ }_{5}^{4}$ ESO Fellow.

${ }^{5}$ School of Physical, Environmental and Mathematical Sciences, University of New South Wales, Australian Defence Force Academy, Canberra, ACT 2600, Australia.

${ }^{6}$ ARC Future Fellow.

${ }^{7}$ Department of Physics, Astronomy and Geosciences, Towson University, Towson, MD, 21252.
}

Although most optical studies have concentrated on the fast-moving O- and Ne-rich ejecta (Lasker 1980, Blair et al. 2000; Vogt \& Dopita 2011), there are a number of luminous, dense shock clouds with apparently 'normal' interstellar composition and $\sim 200 \mathrm{~km} / \mathrm{s}$ velocity dispersion. The most prominent of these is the so-called Lasker's Bowl structure in the northern part of the remnant, but HST imaging (Blair et al. 2000) reveals many other small complexes of shocked cloudlets. These clouds do not show appreciable enhancements in the strength of the [N II] lines, and are thought to be simply ISM clouds over which the SNR blast wave has recently swept. In this paper we follow the study of N49 by Dopita et al. (2016) in investigating the degree of dust destruction in these cloud shocks. We also derive the physical parameters for some 20 different shocked ISM cloudlets and estimate their chemical abundances.

\section{OBSERVATIONS \& DATA REDUCTION}

The integral field spectra of N132D were obtained between 16 Nov 2017 and 24 Nov 2017 using the Wide Field Spectrograph (WiFeS) (Dopita et al. 2007, 2010), an integral field spectrograph mounted on the 2.3-m ANU telescope at Siding Spring Observatory. This instrument delivers a field of view of $25^{\prime \prime} \times 38^{\prime \prime}$ at a spatial resolution of either $1.0^{\prime \prime} \times 0.5^{\prime \prime}$ or $1.0^{\prime \prime} \times 1.0^{\prime \prime}$, depending on the binning on the CCD. In these observations, we operated in the binned $1.0^{\prime \prime} \times 1.0^{\prime \prime}$ mode. The data were obtained in the low resolution mode $R \sim 3000$ (FWHM of $\sim 100 \mathrm{~km} / \mathrm{s}$ ) using the B3000 \& R3000 gratings in each arm of the spectrograph, with the RT560 dichroic which provides a transition between the two arms at around $560 \mathrm{~nm}$. For details on the various instrument observing modes, see Dopita et al. (2007).

All observations are made at $\mathrm{PA}=0$, giving a long axis in the N-S direction. The basic grid consists of 15 pointings in an overlapping $3 \times 5$ grid $(\mathrm{E}-\mathrm{W}: \mathrm{N}-\mathrm{S})$, followed by 
7 pointings centered on the overlap regions of the base $3 \times 5$ grid, with an additional pointing in the SE to probe the extent of the photoionised precursor region around N132D. The eighth overlap position was not observed due to deteriorating weather conditions. The typical seeing over the course of the observations was 1.5 arc sec. and ranged from 0.5 to 2.5 arc sec. over the individual exposures. Each region was observed with $2 \times 1000$ s exposure time giving a total integration time on target of 44000s. We used blind offsets from a reference star to move to each field of the mosaic consistently over the course of the observing run, and avoid any gaps in the resulting mosaic. A fault in the offset guide head gave rise to errors of $\lesssim 5$ arc sec. in the pointing of the fields; small enough not to result in any gaps within the mosaic.

The wavelength scale is calibrated using a series of NeAr arc lamp exposures, taken throughout the night. Arc exposure times are 50s for the B3000 grating and 1s for the R3000 grating. Flux calibration was performed using the STIS spectrophotometric standard stars HD 009051, HD 031128 and HD 0750008 . In addition, a B-type telluric standard HIP 8352 was observed to better correct for the $\mathrm{OH}$ and $\mathrm{H}_{2} \mathrm{O}$ telluric absorption features in the red. The separation of these features by molecular species allows for a more accurate telluric correction by accounting for night to night variations in the column density of these two species. All data cubes were reduced using the PyWiFeS 9 data reduction pipeline (Childress et al. $2014 \mathrm{~b}$ a).

All the individual, reduced cubes were medianaveraged into a red and blue mosaic using a custom PYTHON script. The WCS solution of both mosaics is set by cross-matching their individual (collapsed) white-light images with all entries in the Gaia (Gaia Collaboration et al. 2016a) DR1 (Gaia Collaboration et al. 2016b); see Fig 1). For simplicity, and given the spaxel size of the WiFeS datacubes, we restrict ourselves to integer shifts when combining fields. We estimate that the resulting absolute and relative (field-to-field) alignment accuracy are both \pm 1 arc sec.

\subsection{HST imaging of the cloudlets}

We have downloaded all the HST ACS F475W and F658N images from the Barbara A. Mikulski archive for space telescopes (MAST). The former filter encompasses the full velocity range of the $[\mathrm{O}$ III $] \lambda \lambda 5959,5007 \AA$ emission, whereas the latter covers the rest-frame $\mathrm{H} \alpha$ emission. The dataset comprises four exposures of $380 \mathrm{~s}$ each with the F475W filter, and four exposures of $360 \mathrm{~s}$ each using the F658N filter, all acquired in January 2004 under program \#12001 (PI Green).

We used the individual calibrated and CTE-corrected frames (*_FLC.FITS) retrieved from MAST to construct a combined, drizzled image in both filters using the following steps. We first correct the WCS solutions of each frame using the TWEAKREG routine from the DRIZZLEPAC 2.1.13 package via a dedicated PYTHON script. We anchor the WCS solution to the Gaia (Gaia Collaboration et al. 2016a) DR1 (Gaia Collaboration et al. 2016b) sources present within the images, retrieved au-

8 Available at : WWW.mso. anu.edu. au/ bessell/FTP/Bohlin2013/G012813.html sttp://www.mso.anu.edu.au/pywifes/doku.php.
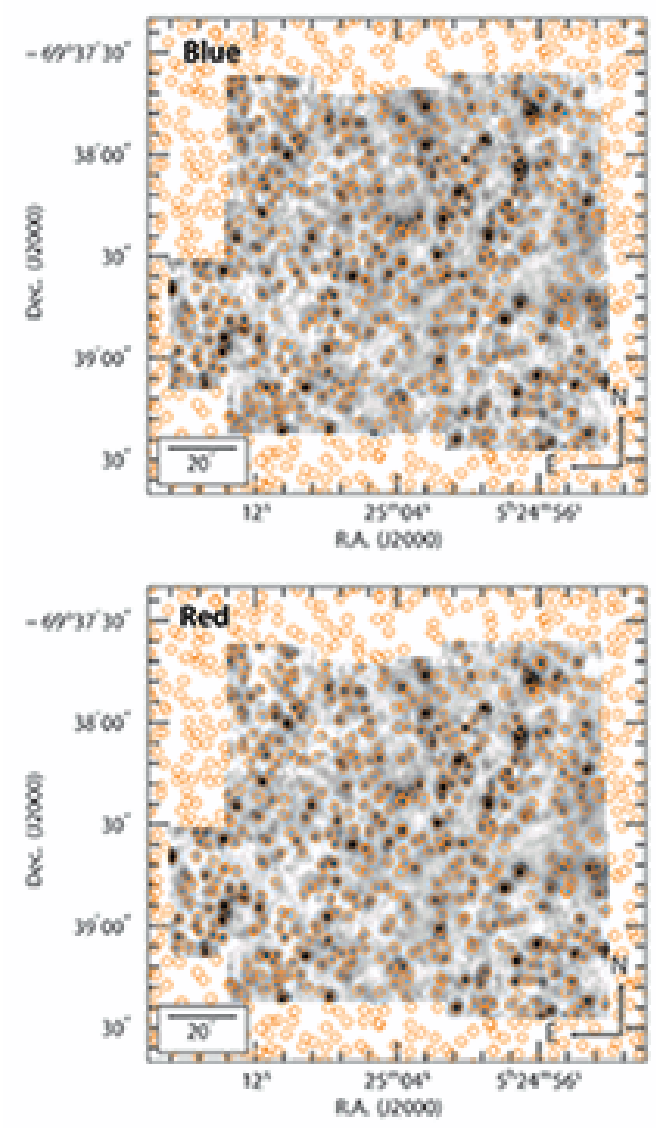

FIG. 1. - The alignment charts showing the reconstructed mosaic in the summed 5600-5680 $\AA$ wavelength region for the blue arm (above) and the red arm (below). For reference, the positions of all the GAIA entries in the area are shown as orange circles.

tomatically via the ASTROQUERY module. The WCScorrected images are then merged using the ASTRODRIZZLE routine, with a pixel scale set to 0.04 arcsec for both filters (for simplicity).

\subsection{Spectral Extraction}

The spectra of 20 prominent shocked ISM clouds were extracted from the global WiFeS mosaic datacubes using using QFitsView v3.1 rev.741 ${ }^{10}$ The positions of the clouds were chosen so as to largely avoid contamination by high-velocity [O III] - emitting material, see Fig 2. The exception is P07, but the velocity shift of the high velocity material here is sufficient as to allow accurate determinations of the narrow-line fluxes. We used a circular extraction aperture with a radius of either 2 or 3 arc sec. to best match the size of the bright region. To remove the residual night sky emission and (approximately) the faint stellar contribution, we subtracted a mean sky reference annulus 1 arc sec. wide surrounding the extraction aperture. The extraction regions were optimised by peaking up the signal in $\mathrm{H} \alpha$ in the red data cube, and in $\mathrm{H} \gamma$ in the blue data cube, respectively. This procedure may lead to some contamination by the faint

\footnotetext{
10 QFitsView v3.1 is a FITS file viewer using the QT widget library and was developed at the Max Planck Institute for Extraterrestrial Physics by Thomas Ott.
} 


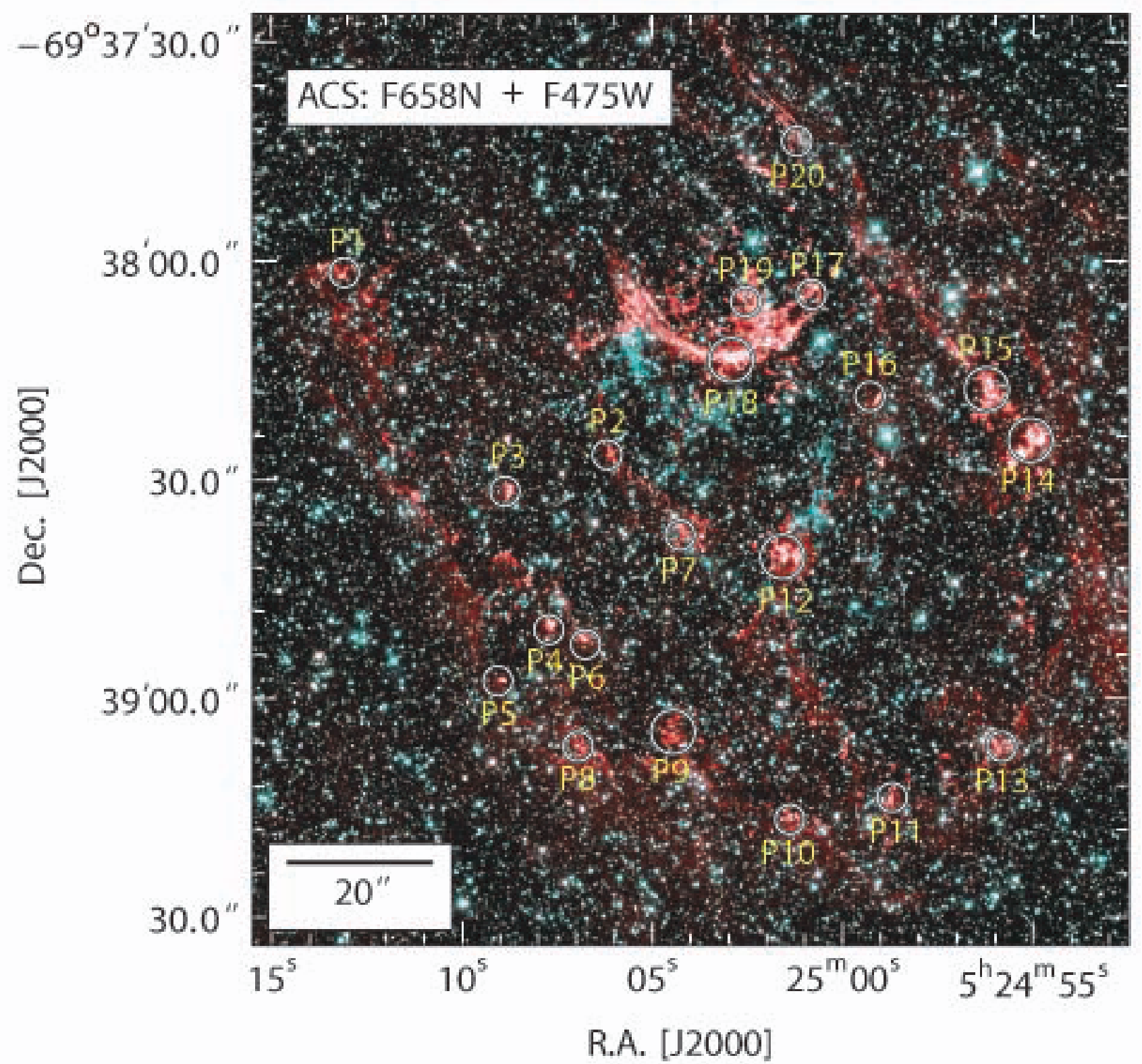

FIG. 2.- The shocked cloud regions listed in Table 1 are here displayed on an HST H $\alpha+[\mathrm{O}$ III] image. The fast-moving [O III] clouds appear as bright turquoise regions. Note that P20 is particularly strong in [O III], due to the fact that the cloud shock here has not yet become fully radiative.

extended cloud emission in the case of P07, P08, P14, P15, P18 and P20 (see Figure 3). However, the core region strongly dominates such fainter contributions. The positions of the extracted spectra, the extraction radius used, the measured mean radial velocity, and the $\mathrm{H} \alpha$ velocity width (FWHM) (after correction for the instrumental resolution) of each cloud are listed in Table 1, and the corresponding positions are shown on the HST ACS $\mathrm{H} \alpha+[\mathrm{O}$ III] image in Figure 2 and each cloud with its spectral extraction region is shown in an $8 \times 8$ arc sec. postage stamp image in Figure 3. A spectrum of the bright cloudlet P14 is shown in Figure 4 to indicate the quality of the data. Note that the typical velocity FWHM of these clouds in only $\sim 180 \mathrm{~km} / \mathrm{s}$, which is fairly indicative of the mean shock velocity in those clouds (Fig.6 in Dopita et al. 2012).

The mean Heliocentric radial velocity of all 20 clouds is $+246 \mathrm{~km} / \mathrm{s}$, and the mean Heliocentric radial velocity of the photoionised halo of N132D is found to be $+261 \mathrm{~km} / \mathrm{s}$, a difference which lies within the expected statistical error caused by sampling of the cloud shock velocities projected along the lines of sight within the random cloud positions inside the SNR. These figures can be compared to the $+264.6 \mathrm{~km} / \mathrm{s}$ (Smith \& Weedman $1971),+262 \pm 16 \mathrm{~km} / \mathrm{s}$ (Danziger \& Dennefeld 1976), and $+259 \mathrm{~km} / \mathrm{s}$ (Feast 1964) for N127, a nearby HII region.

Since most of the $\mathrm{H} \alpha$ emission arises in the recombination zone of the cloud shocks, we can estimate a 

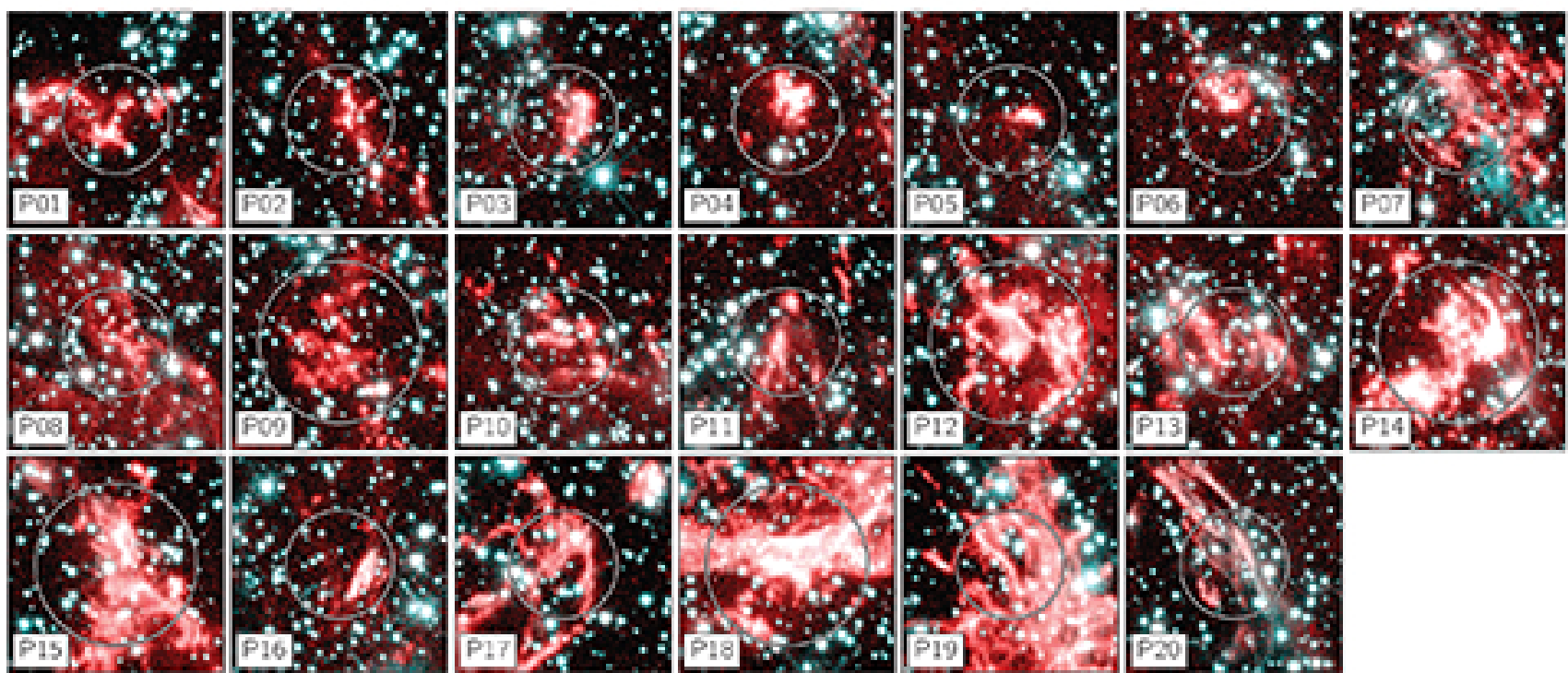

FIG. 3. - HST postage-stamp images of the 20 ISM clouds studied in this paper. Each image covers $8 \times 8$ arc sec. on the sky, or 1.78 pc at the distance of the LMC. The extraction apertures used for the spectra are shown as circles.

TABLE 1

The POSITIONS, EXTRACTION APERTURE SIZES, AND VELOCITIES OF THE SHOCKED INTERSTELLAR CLOUDS OF N132D

\begin{tabular}{cccccc}
\hline \hline $\begin{array}{c}\text { Posn. } \\
\#\end{array}$ & $\begin{array}{c}\text { RA } \\
(\mathrm{J} 2000)\end{array}$ & $\begin{array}{c}\text { Dec } \\
(\mathrm{J} 2000)\end{array}$ & $\begin{array}{c}\text { Ap. Diam. } \\
(\text { arc sec. })\end{array}$ & $\begin{array}{c}V_{\text {Hel. }} \\
v_{\text {Hel }}(\mathrm{km} / \mathrm{s})\end{array}$ & $\begin{array}{c}\Delta V_{\mathrm{FWHM}} \\
(\mathrm{km} / \mathrm{s})\end{array}$ \\
\hline P01 & $05: 25: 13.085$ & $-69: 38: 01.6$ & 4 & 312 & 190 \\
P02 & $05: 25: 06.188$ & $-69: 38: 26.6$ & 4 & 202 & 190 \\
P03 & $05: 25: 08.870$ & $-69: 38: 31.6$ & 4 & 273 & 203 \\
P04 & $05: 25: 07.720$ & $-69: 38: 50.6$ & 4 & 434 & 159 \\
P05 & $05: 25: 09.062$ & $-69: 38: 57.6$ & 4 & 256 & 216 \\
P06 & $05: 25: 06.762$ & $-69: 38: 52.6$ & 4 & 333 & 159 \\
P07 & $05: 25: 04.271$ & $-69: 38: 37.6$ & 4 & 204 & 201 \\
P08 & $05: 25: 06.953$ & $-69: 39: 06.6$ & 4 & 321 & 136 \\
P09 & $05: 25: 04.460$ & $-69: 39: 04.6$ & 6 & 259 & 155 \\
P10 & $05: 25: 01.391$ & $-69: 39: 16.6$ & 4 & 333 & 178 \\
P11 & $05: 24: 58.707$ & $-69: 39: 13.6$ & 4 & 373 & 173 \\
P12 & $05: 25: 01.587$ & $-69: 38: 40.6$ & 6 & 186 & 204 \\
P13 & $05: 24: 55.833$ & $-69: 39: 06.6$ & 4 & 321 & 150 \\
P14 & $05: 24: 55.074$ & $-69: 38: 24.6$ & 6 & 402 & 183 \\
P15 & $05: 24: 56.225$ & $-69: 38: 17.6$ & 6 & 321 & 198 \\
P16 & $05: 24: 59.291$ & $-69: 38: 18.6$ & 4 & 231 & 178 \\
P17 & $05: 25: 00.823$ & $-69: 38: 04.6$ & 4 & 214 & 158 \\
P18 & $05: 25: 02.932$ & $-69: 38: 13.6$ & 6 & 216 & 222 \\
P19 & $05: 25: 02.549$ & $-69: 38: 05.6$ & 4 & 118 & 152 \\
P20 & $05: 25: 01.211$ & $-69: 37: 43.6$ & 4 & 330 & 152 \\
\hline
\end{tabular}

lower limit for the cloud shock velocities, $v_{s}$, from the most extreme differences in the measured radial velocity of the cloud from the mean Heliocentric radial velocity of the photoionised halo of N132D. These are $+173 \mathrm{~km} / \mathrm{s}$ for cloud P04, and $-143 \mathrm{~km} / \mathrm{s}$ for cloud P19. Thus we have $v_{s} \gtrsim 160 \mathrm{~km} / \mathrm{s}$, in good agreement with the estimate based on the FWHM of the $\mathrm{H} \alpha$ line profiles.

\subsection{Measuring Emission Line Fluxes}

For each extracted spectrum, the spectra were first reduced to rest wavelength based on their measured radial velocities listed in Table 1, and then emission-line fluxes in units of $\mathrm{erg} / \mathrm{cm}^{2} / \mathrm{s}$, their uncertainties, the emission line FWHMs (in $\AA$ ) and the continuum levels were measured using the interactive routines in Graf
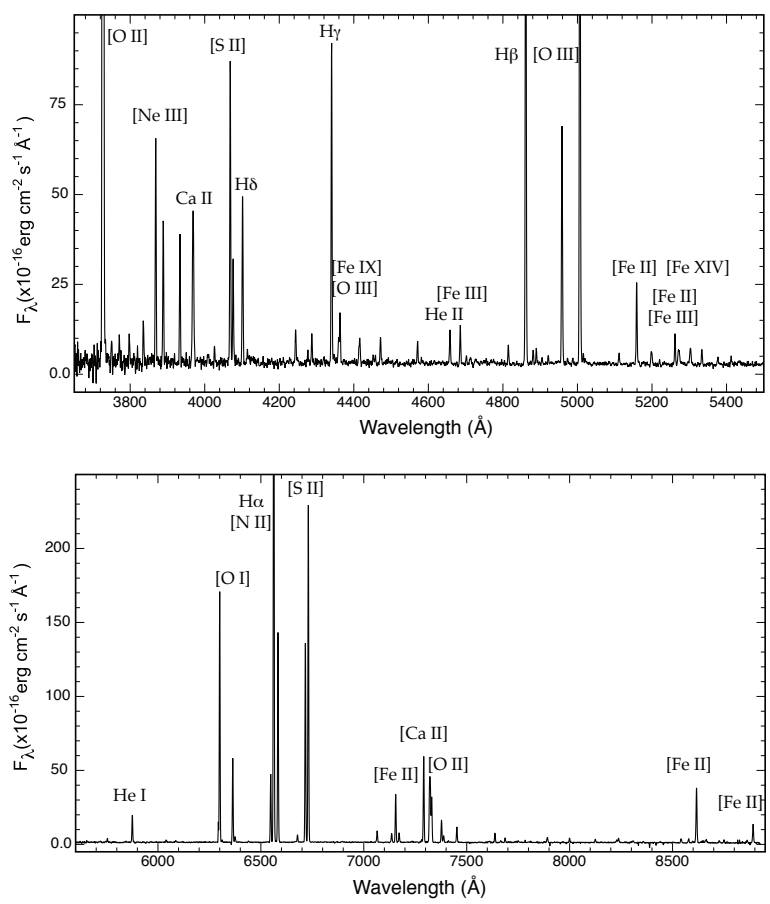

FIG. 4. - The extracted spectrum of the bright cloud P14 to show the quality of the data. Note the absence of both night sky emission lines, detectable atmospheric absorption, and underlying stellar continuum. The more prominent emission lines in the spectrum are identified according to ionic species. 
11 and in Lines 12 . The measured line fluxes relative to $\mathrm{H} \beta$ and their uncertainties along with the absolute $\mathrm{H} \beta$ fluxes in units of $\mathrm{erg} / \mathrm{cm}^{2} / \mathrm{s}$, are given for each of the clouds in Tables A1- A4 in the Appendix.

\section{EMISSION LINE DIAGNOSTICS}

\subsection{Self-Consistent Shock Modelling}

To analyse the spectrophotometry we have built a family of radiative shocks with self-consistent pre-ionisation using the MAPPINGS 5.12 code, following the methodology described in Sutherland \& Dopita (2017), and applied to the study of Herbig-Haro objects by Dopita \& Sutherland (2017). The pre-shock density is set by the ram pressure of the shock, taken to be independent of shock velocity; $P_{\text {ram }}=1.5 \times 10^{-7}$ dynes $\mathrm{cm}^{-2}$, to ensure that the measured $[\mathrm{S} \mathrm{II}]$ densities (which give the electron density near the recombination zone of the shock) approximately match those produced by the models. For each abundance set, a set of models was run for $100 \leqslant v_{s} \leqslant 475 \mathrm{~km} / \mathrm{s}$ in steps of $25 \mathrm{~km} / \mathrm{s}$. The magnetic field pressure in the un-shocked cloud ahead of the photo-ionised precursor is assumed to be in equipartition with the gas pressure, $P_{\mathrm{mag}}=P_{\text {gas }}$, and the temperature of the gas entering the shock is given by the selfconsistent pre-ionisation computation; see Sutherland \& Dopita (2017) for details.

The abundance set was initially taken as being 0.5 times the Local Galactic Concordance (LGC) values (Nicholls et al. 2017), but was then iterated manually to achieve a better fit of theory to the spectra of the clouds on the standard Baldwin et al. (1981) and Veilleux \& Osterbrock (1987) diagnostic diagrams. The depletion factors of the heavy elements caused by the condensation of these elements onto dust are defined as the ratio of the gas phase abundance to the total element abundance. The depletion factors are derived from the formulae of Jenkins (2009), extended to the other elements on the basis of their condensation temperatures and/or their position on the periodic table. In these shock models, following Dopita et al. (2016) we have investigated the effect of changing the logarithmic Fe depletion, $\log D_{\mathrm{Fe}}$ in the range $0.0>\log \left[D_{\mathrm{Fe}}\right]>-1.0$.

The abundance set adopted for the theoretical grid at the various values of $\log D_{\mathrm{Fe}}$ is given in Table 2. The abundances of $\mathrm{C}, \mathrm{Mg}$, and $\mathrm{Si}$ are not constrained by our observations and for these we fix the abundances at half of the LGC values. We produce a more refined estimate of the LMC chemical abundances when we build detailed models for the brightest clouds, below.

The line intensities and line ratios given in this paper comprise the sum of the radiative shock and its photoionised precursor. In the case of the fastest shock, the extent of the photoionised precursor region and/or the cooling length of the shock may exceed the physical extent of the pre-shocked cloud. In Figure 5 we show the cooling length of the shock to $1000 \mathrm{~K}$, the depth of the photoionised precursor to the point where hydrogen is only $1 \%$ ionised, and the fraction of the shock $\mathrm{H} \beta$ emission which arises from the precursor. The cooling

11 Graf is written by R. S. Sutherland and is available at: https: $/ /$ miocene.anu.edu.au/graf

${ }^{12}$ Lines is written by R. S. Sutherland and is available at: https: $/ /$ miocene.anu.edu.au/lines
TABLE 2

THE LMC ABUNDANCE SET USED IN THE GRID, AND THE CORRESPONDING GAS PHASE ABUNDANCES FOR VARIOUS LOGARITHMIC DEPLETION FACTORS IN FE.

\begin{tabular}{l|ccccc}
\hline \hline & \multicolumn{5}{|c|}{$12+\log (\mathrm{X} / \mathrm{H})$} \\
\cline { 2 - 6 } Element & Total & $\log D_{\mathrm{Fe}}$ & $\log D_{\mathrm{Fe}}$ & $\log D_{\mathrm{Fe}}$ & $\log D_{\mathrm{Fe}}$ \\
& Abund. & -0.25 & -0.50 & -0.75 & -1.00 \\
\hline $\mathrm{H}$ & 12.00 & 12.00 & 12.00 & 12.00 & 12.00 \\
$\mathrm{He}$ & 10.96 & 10.96 & 10.96 & 10.96 & 10.96 \\
$\mathrm{C}$ & 8.01 & 7.99 & 7.97 & 7.95 & 7.93 \\
$\mathrm{~N}$ & 7.30 & 7.30 & 7.30 & 7.30 & 7.30 \\
$\mathrm{O}$ & 8.25 & 8.25 & 8.25 & 8.24 & 8.23 \\
$\mathrm{Ne}$ & 7.79 & 7.79 & 7.79 & 7.79 & 7.79 \\
$\mathrm{Mg}$ & 7.26 & 7.26 & 7.25 & 7.15 & 6.95 \\
$\mathrm{Si}$ & 7.20 & 7.20 & 7.20 & 7.15 & 6.93 \\
$\mathrm{~S}$ & 7.00 & 7.00 & 7.00 & 7.00 & 7.00 \\
$\mathrm{Cl}$ & 5.20 & 5.20 & 5.20 & 5.20 & 5.20 \\
$\mathrm{Ar}$ & 5.90 & 5.90 & 5.90 & 5.90 & 5.90 \\
$\mathrm{Ca}$ & 6.02 & 6.02 & 5.66 & 5.27 & 4.87 \\
$\mathrm{Fe}$ & 7.22 & 6.97 & 6.72 & 6.47 & 6.22 \\
$\mathrm{Ni}$ & 5.90 & 5.78 & 5.49 & 5.19 & 4.91 \\
\hline
\end{tabular}

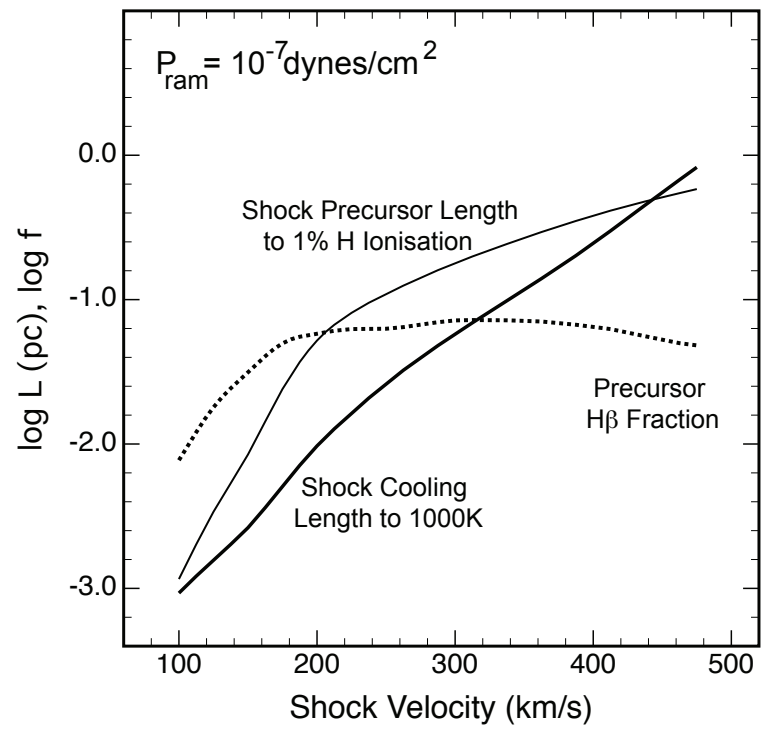

FIG. 5.- The computed cooling length of the shock to $T_{e}=$ $1000 \mathrm{~K}$ (thick line), the thickness of the photoionised precursor to the point where hydrogen is only $1 \%$ ionised (thin line), and the fraction of the shock $\mathrm{H} \beta$ emission which arises from the precursor (dotted line). These are all given for a ram pressure of $P_{\text {ram }}=1.0 \times 10^{-7}$ dynes $\mathrm{cm}^{-2}$. At the densities of those models, the shock cooling length scales inversely as the ram pressure, while the precursor length is almost unaffected by changes in the ram pressure.

length and precursor length are given for a ram-pressure of $P_{\text {ram }}=1.0 \times 10^{-7}$ dynes $\mathrm{cm}^{-2}$. The shock cooling length scales inversely as the ram pressure, and the precursor length remains approximately constant. At the distance of the LMC $0.1 \mathrm{pc}$ corresponds to 0.45 arc sec.

\subsection{Shock Velocity Diagnostics}

Here we investigate potentially useful diagnostics of the shock velocity using emission line ratios. To avoid issues of chemical abundance difference, the line ratio used should involve different levels of the same ion, or else dif- 

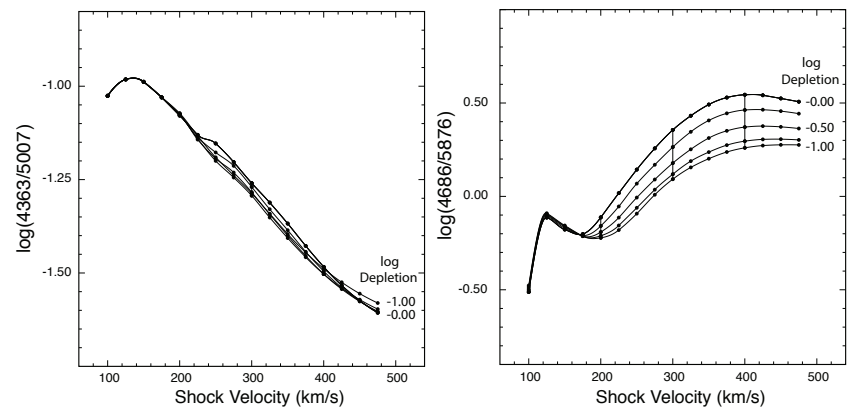

FIG. 6.- The shock velocity sensitive line ratios $\left[\begin{array}{ll}\mathrm{O} & \mathrm{III}\end{array}\right]$ $\lambda \lambda 4363 / 5007$ (left) and He II/He I $\lambda \lambda 4686 / 5876$ (right) as a function of shock velocity and iron depletion factor; $\log D_{\mathrm{Fe}}$.

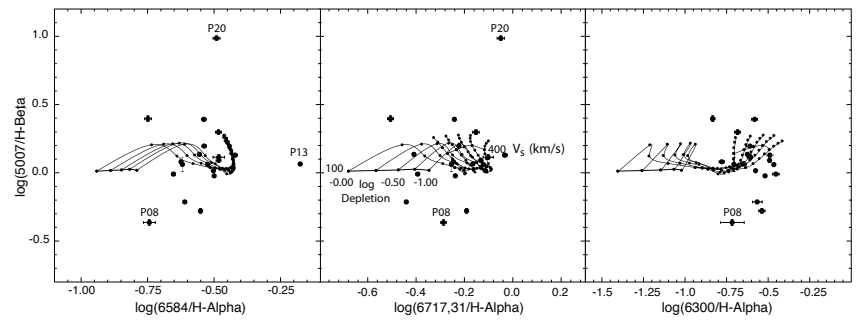

FIG. 7.- The standard BPT plots showing the positions of our clouds superimposed on the theoretical grid. Overall, the model grid reproduces the observations in a satisfactory manner. Anomalous regions are indicated. These are discussed further in Section 4

ferent ions of the same element. In Dopita et al. (2016), we already noted that the [O III] $\lambda \lambda 4363 / 5007$ ratio was a useful indicator of shock velocity. This is because the [O III] temperature in the cooling zone of the shock is high at low shock velocities (faster than $\sim 85 \mathrm{~km} / \mathrm{s}$, when this ion is first produced in the cooling zone of the shock), reaching a maximum at $v_{s} \sim 140 \mathrm{~km} / \mathrm{s}$ when O III starts to become ionised to higher ionisation stages. As the velocity increases further, $\mathrm{O}$ III is ionised to O IV, and the $[\mathrm{O}$ III $]$ emission becomes confined to the cooler region nearer the recombination zone of the shock. At still higher velocities, a O III zone photoionised by EUV photons generated in the cooling zone of the shock develops adjacent to the recombination zone. This has still lower mean temperature; $T_{e} \lesssim 10^{4} \mathrm{~K}$. For these physical reasons, the [O III] temperature is more or less a decreasing function of shock velocity, and is relatively insensitive to $\log D_{\mathrm{Fe}}$. This behaviour is shown in Figure 6.

A second diagnostic is the excitation of $\mathrm{He}$, as measured by the He II/He I $\lambda \lambda 4686 / 5876$ ratio. This is shown in the second panel of Figure 6. Below $v_{s} \sim$ $200 \mathrm{~km} / \mathrm{s}$, this ratio is determined by the pre-ionisation of the shock, and the temperature structure of the postshock region, and is multi-valued. However, above this velocity, it provides a useful diagnostic, albeit somewhat sensitive to $\log D_{\mathrm{Fe}}$. This sensitivity is caused by changes in the cooling length with gas-phase Fe abundance, which changes the ionisation structure of $\mathrm{He}$ in the shock. In what follows, we will use both the He and [O III] diagnostic ratios as spectroscopic indicators of the shock velocity.

\subsection{BPT diagnostic diagrams}

For shocks, the position of the observations on the standard Baldwin et al. (1981) and Veilleux \& Oster-
TABLE 3

SHOCK VELOCITIES DERIVED FROM THE [O III] LINE RATIO AND ESTIMATED FE II DEPLETION FACTORS OF THE SHOCKED INTERSTELLAR CLOUDS OF N132D

\begin{tabular}{|c|c|c|c|c|c|}
\hline Line: & & {$\left[\begin{array}{l}\text { Fe II }] \\
\end{array}\right.$} & & {$[\mathrm{Fe} \mathrm{II}]$} & \\
\hline $\begin{array}{l}\text { Posn. } \\
\#\end{array}$ & $\begin{array}{c}V_{\text {OIII }} \\
(\mathrm{km} / \mathrm{s})\end{array}$ & $\begin{array}{r}\lambda \mathbf{5 1 5 8} \\
\log D_{\mathrm{Fe}} \\
\end{array}$ & Err. & $\begin{array}{r}\lambda \mathbf{8 6 1 7} \\
\log D_{\mathrm{Fe}} \\
\end{array}$ & Err. \\
\hline P02 & $190 \pm 40$ & 0.00 & 0.03 & -0.05 & 0.02 \\
\hline P03 & $225 \pm 50$ & -0.13 & 0.07 & -0.12 & 0.03 \\
\hline P04 & $200 \pm 50$ & 0.20 & 0.05 & 0.10 & 0.02 \\
\hline P05 & $325 \pm 35$ & -0.19 & 0.06 & -0.09 & 0.05 \\
\hline P06 & $\ldots$ & 0.00 & 0.05 & 0.00 & 0.05 \\
\hline P07 & $230 \pm 70$ & -0.10 & 0.05 & -0.15 & 0.02 \\
\hline P10 & $210 \pm 40$ & -0.42 & 0.10 & -0.38 & 0.05 \\
\hline P11 & $285 \pm 35$ & -0.32 & 0.12 & -0.10 & 0.03 \\
\hline $\mathrm{P} 12$ & $260 \pm 25$ & -0.13 & 0.02 & -0.23 & 0.03 \\
\hline $\mathrm{P} 13$ & $250 \pm 40$ & -0.02 & 0.03 & -0.20 & 0.03 \\
\hline P14 & $280 \pm 20$ & -0.11 & 0.02 & -0.02 & 0.02 \\
\hline P15 & $240 \pm 25$ & -0.13 & 0.02 & -0.15 & 0.02 \\
\hline P16 & $210 \pm 50$ & -0.40 & 0.06 & -0.32 & 0.07 \\
\hline P17 & $210 \pm 40$ & -0.25 & 0.09 & & \\
\hline P18 & $360 \pm 20$ & -0.15 & 0.02 & -0.12 & 0.02 \\
\hline P19 & $310 \pm 40$ & -0.27 & 0.04 & & \\
\hline P20 & $190 \pm 20$ & 0.00 & 0.12 & -0.30 & 0.10 \\
\hline Av: & 248 & -0.14 & & -0.14 & \\
\hline
\end{tabular}

brock (1987) diagnostic diagrams (hereafter BPT Diagrams), is indicative of the mode of excitation. However, they are not really useful as diagnostics of the detailed shock conditions. This is demonstrated in Figure 7. It should be noted that the range in observed line ratio and in the theoretical grid is very small on these diagrams, compared with the range covered by either HII regions or active galaxies and Narrow Line Regions. All these diagrams serve to show is that the theoretical models and the observations show a satisfactory overlap.

This said, a few points on these diagrams are worthy of further mention. First P13 is the only region which shows an excess in the line intensity of [N II], which may be indicative of pre-supernova mass-loss enrichment. However, this seems a little unlikey as the region is close to the boundary of the SNR shell. Second, P20 shows extremely strong [O III] emission. This is evidenced by the blue appearance of this region in Figure 3 . This region will be discussed in more detail below, where we show that it is due to a finite-age shock in which the gas has not had time to become fully radiative. Lastly, P08 shows particularly weak [O III] emission. This region, also discussed below, lies in a complex of Balmer dominated or non-radiative filaments in which a high velocity non-radiative shock is passing through an un-ionised or partially-ionised precursor medium.

\section{4. [Fe II], [Fe III] and [Fe VII] diagnostics}

As can be seen from Tables A1- A4 the [Fe II] spectrum is very rich. For the purposes of analysis, we have selected the brightest of these lines, [Fe II] $\lambda 5158$ and $[$ Fe II $] \lambda 8617$. In Figure 8 we present the observed points superimposed on our theoretical shock model grid. From this, it is immediately apparent that nowhere does the measured iron depletion factor; $\log D_{\mathrm{Fe}}$ exceed -0.5. Indeed many of the points are consistent with no depletion at all. 

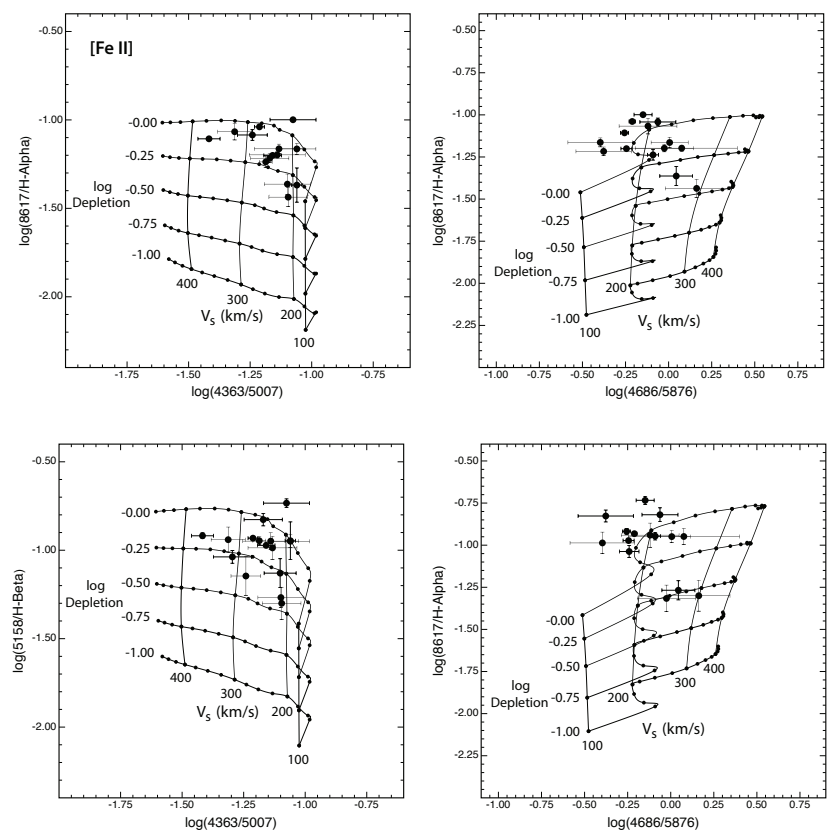

FIG. 8. - The depletion of iron as measured by the strong [Fe II] lines. The data are consistent with a mean depletion factor $\log D_{\mathrm{Fe}} \sim-0.14$
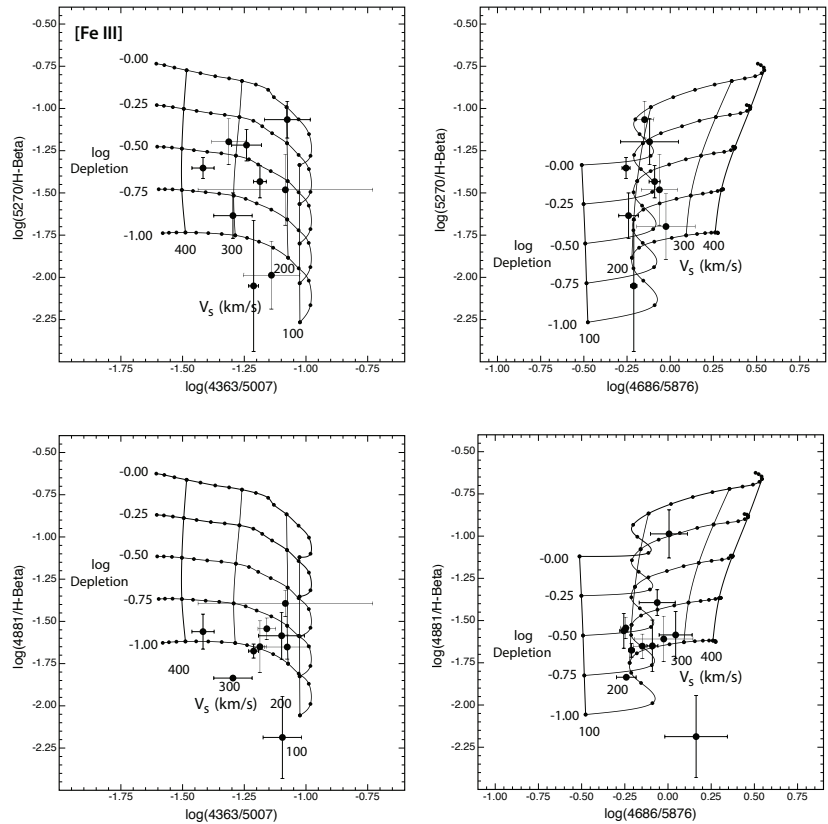

FIG. 9. - The depletion of iron using the strong [Fe III] lines. The data are consistent with a mean depletion factor $\log D_{\mathrm{Fe}} \sim-0.74$

Furthermore, it is clear that the indicated shock velocities in general exceed $200 \mathrm{~km} / \mathrm{s}$. Thus for most of these the [O III] line ratio can be used to estimate the shock velocity. However, a number of the points lie in the ambiguous region of the $\mathrm{He}$ II/He I line ratio velocity diagnostic. To the extent to which it is possible, both shock velocities and $\log D_{\mathrm{Fe}}$ have been estimated for each cloud from these diagnostic diagrams. The results are given in Tables 3 and 4 We find a mean shock velocity $\left\langle v_{s}\right\rangle$ of $240 \mathrm{~km} / \mathrm{s}$, and $\left\langle\log D_{\mathrm{Fe}}\right\rangle=-0.15$. This result agrees very
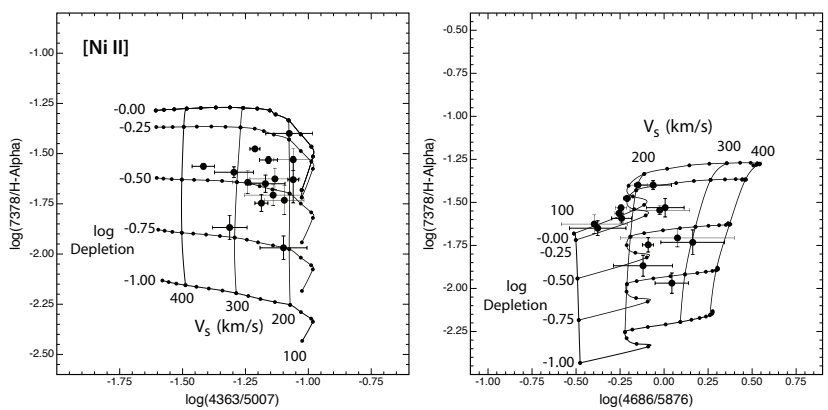

FIG. 10. - The depletion of Fe using the $[\mathrm{Fe}$ VII $] \lambda 6087 / \mathrm{H} \alpha$ ratio. The data are consistent with a mean depletion factor $\log D_{\mathrm{Fe}} \sim$ -0.4 and a range of $\sim-0.0--0.75$.
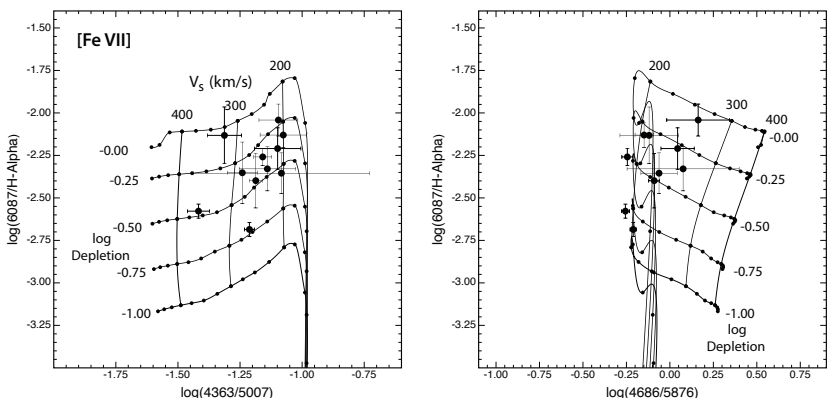

FIG. 11. - The depletion of $\mathrm{Ni}$ using the $[\mathrm{Ni}$ II $] \lambda 7378 / \mathrm{H} \alpha$ ratio. The data are consistent with a mean depletion factor $\log D_{\mathrm{Fe}} \sim$ -0.4 and a range of $\sim-0.0--0.75$.
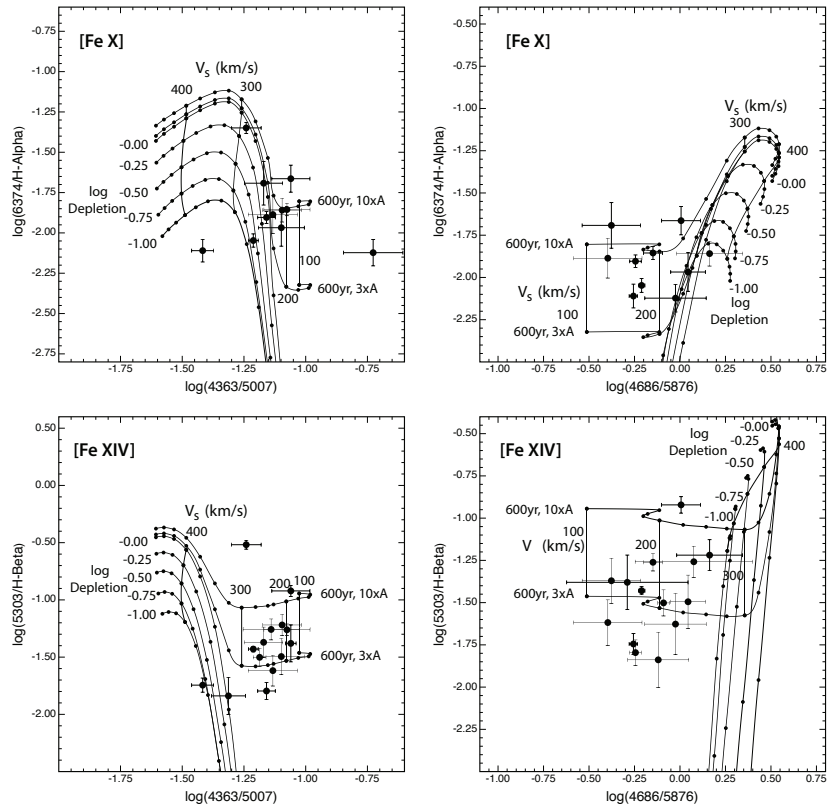

FIG. 12.- The $[\mathrm{Fe} \mathrm{X}] \lambda 6374 / \mathrm{H} \alpha$ and $[\mathrm{Fe} \mathrm{XIV}] \lambda 5303 / \mathrm{H} \beta$. The standard shock grid, as presented in the previous figures clearly fails to reproduce the positions of the observed points on these diagnostic diagrams. However, by adding a faster, partially radiative shock of age $\sim 600 \mathrm{yr}$, which covers an area of 3-10 times the area of the dense cloud shock, we can achieve a satisfactory fit with the observations (upper two curves marked 600yr, 3xA and 600yr, 10xA, respectively). 
TABLE 4

SHock Velocities DeRIVED FROM THE He II/He I LiNe RATIO AND ESTIMATED FE II DEPLETION FACTORS OF THE SHOCKED INTERSTELLAR CLOUDS OF N132D

\begin{tabular}{lccccc}
\hline \hline Line: & & {$[\mathbf{F e ~ I I}]$} & \multicolumn{3}{c}{$[\mathbf{F e ~ I I}]$} \\
Posn. & $V_{\mathrm{He}}$ & $\lambda \mathbf{5 1 5 8}$ & & $\lambda \mathbf{8 1 7}$ \\
$\#$ & $(\mathrm{~km} / \mathrm{s})$ & $\log D_{\mathrm{Fe}}$ & Err. & $\log D_{\mathrm{Fe}}$ & Err. \\
\hline P01 & $225 \pm 55$ & -0.50 & 0.10 & -0.15 & 0.03 \\
P02 & $225 \pm 25$ & -0.10 & 0.04 & -0.10 & 0.03 \\
P03 & $\ldots$ & $\ldots$ & & -0.10 & 0.05 \\
P04 & $200 \pm 25$ & 0.20 & 0.05 & 0.10 & 0.02 \\
P05 & $200 \pm 50$ & -0.03 & 0.06 & 0.02 & 0.02 \\
P06 & $210 \pm 24$ & 0.08 & 0.05 & 0.03 & 0.03 \\
P07 & $245 \pm 100$ & -0.12 & 0.05 & -0.20 & 0.02 \\
P10 & $300 \pm 75$ & -0.55 & 0.15 & -0.48 & 0.05 \\
P12 & $210 \pm 20$ & -0.07 & 0.02 & -0.20 & 0.04 \\
P14 & $175 \pm 15$ & 0.08 & 0.02 & 0.12 & 0.02 \\
P15 & $170 \pm 20$ & 0.05 & 0.02 & -0.10 & 0.02 \\
P16 & $265 \pm 30$ & -0.49 & 0.08 & -0.35 & 0.06 \\
P18 & & & & 0.00 & 0.03 \\
P20 & & & & -0.27 & 0.10 \\
& & & & & \\
\hline Av: & 220 & -0.13 & & -0.12 & \\
\hline
\end{tabular}

closely with the $\left\langle\log D_{\mathrm{Fe}}\right\rangle=-0.16 \pm 0.07$ found by Dopita et al. (2016) in N49, another bright LMC SNR, and shows that dust has been largely destroyed by the time the recombination zone of the shock has been reached.

A similar analysis was applied to the $[\mathrm{Fe} \mathrm{III}] \lambda 5270 / \mathrm{H} \beta$ and $[\mathrm{Fe} \mathrm{III}] \lambda 4881 / \mathrm{H} \beta$ line ratios. This is shown in Figure 9. The two lines agree less well with each other, the $[\mathrm{Fe}$ III $] \lambda 4881 / \mathrm{H} \beta$ ratio tending to produce a larger depletion factor. In each line ratio, a much wider range of depletion factors is observed, from -0.25 to $\sim-1.25$. Averaging all measurements, we obtain $\left\langle\log D_{\mathrm{Fe}}\right\rangle=-0.74 \pm$ 0.25 . This result is also broadly consistent with what was derived in the case of $\mathrm{N} 49 ;\left\langle\log D_{\mathrm{Fe}}\right\rangle=-0.956 \pm 0.15$ (Dopita et al. 2016).

Finally, the analysis for $[\mathrm{Fe} \mathrm{VII}] \lambda 6087 / \mathrm{H} \alpha$ is shown in Figure 11. Note that this line requires shock velocities of $v_{s}>180 \mathrm{~km} / \mathrm{s}$ in order to be produced with appreciable strength in the radiative shock. Here, the inferred depletion factors range from -0.0 to $\sim-0.75$ with $\left\langle\log D_{\mathrm{Fe}}\right\rangle=-0.4$.

Given the intrinsic uncertainties in the atomic data for the forbidden lines of Fe, we can conclude that the [Fe III $]$ and the [Fe VII] lines imply a mean depletion factor of order $\left\langle\log D_{\mathrm{Fe}}\right\rangle=-0.6 \pm 0.25$, while the [Fe II] lines give a depletion factor $\left\langle\log D_{\mathrm{Fe}}\right\rangle=-0.15 \pm 0.15$. Thus, in both of these N132D and in N49, we find that Fe-bearing dust grains are not fully destroyed until they reach the recombination zone of the shock. The detail of the grain destruction process were discussed in Dopita et al. (2016), where it was concluded that this type of depletion pattern supports the grain destruction model of Seab \& Shull (1983) and Borkowski \& Dwek (1995).

\section{5. [Ni II] diagnostics}

Since we also observe strong $[\mathrm{Ni}$ II $] \lambda 7378$, it is worth asking whether the Ni-containing grains suffer the same fate as the Fe-containing grains. The diagnostic plots for $[\mathrm{Ni} \mathrm{II}] / \mathrm{H} \alpha$ are given in Figure 10 . We find a depletion factor of $\sim-0.4$, intermediate between the [Fe III] and [Fe III] results, but again consistent with a large fraction of dust grains being destroyed in the shock.

\subsection{The highly ionised species of Fe}

To produce the highly ionised species of Fe; $[\mathrm{Fe}$ IX] $\lambda 8235,[\mathrm{Fe} \mathrm{X}] \lambda 6374$ and $[\mathrm{Fe} \mathrm{XIV}] \lambda 5303$ requires shock velocities $v_{s} \gtrsim 200 \mathrm{~km} / \mathrm{s}$. Indeed, the selfconsistent radiative shock models do not produce strong [Fe X] $\lambda 6374$ until $v_{s} \sim 250 \mathrm{~km} / \mathrm{s}$ and [Fe XIV] $\lambda 5303$ becomes bright only for $v_{s}>350 \mathrm{~km} / \mathrm{s}$. The diagnostics of expansion velocity, velocity dispersion, and the spectral diagnostics presented above all point to shock velocities for typical clouds in the range $160 \mathrm{~km} / \mathrm{s}<v_{s}<300 \mathrm{~km} / \mathrm{s}$. Thus it is clear that the relatively strong $[\mathrm{Fe} \mathrm{X}]$ and [Fe XIV] lines observed must arise in another region with higher shock velocity.

This is not unexpected, given the appearance of the clouds on the HST images, which often show a bright core in $\mathrm{H} \alpha$, surrounded by fainter filamentary structures. We can identify these regions with the bright radiative shocks being driven into the denser cores of these clouds, and a faster, but only partially-radiative shock driven by a similar external ram-pressure sweeping through the less dense outer regions of the same clouds. Indeed in the case of another SNR in the LMC; N49, both the spatial and dynamical distinction between the [Fe XIV] $\lambda 5303$ and the $[\mathrm{Fe}$ II] $] 55159$ emission are very marked (cf. Fig.3 in Dopita et al. 2016).

The detailed diagnostic diagrams for these two $\mathrm{Fe}$ species are shown in Figure 12. For the standard grid, both fail to reproduce the observed strength of the lines, but the [Fe XIV] is particularly bad. Introducing a partially-radiative shock leads to a dramatic improvement. The derivation of the plausible shock parameters for these shocks is as follows.

First, we note that the mean cloud shock ram pressure derived from the observations (see Section 4 . below) is $P_{\text {ram }}=3.3 \times 10^{-7}$ dynes $/ \mathrm{cm}^{2}$. At the mean pre-shock particle density implied for these shocks, $n_{0} \sim 240 \mathrm{~cm}^{-3}$, the mean cooling age to $T_{e}=1000 \mathrm{~K}$ is $400 \mathrm{yr}$. This provides an initial estimate of the shock age. Another way is to take the age of the SNR as estimated from the [O III] dynamics of the fast-moving material and estimate from the position of the cloud within the SNR how long it has been since the blast wave overran the cloud. In Section 1 we saw that estimates of the age of N132D vary from 1350 - 3440yr for the SNR, with a probable age of $\sim 2500 \mathrm{yr}$ (Danziger \& Dennefeld 1976, Lasker 1980, Vogt \& Dopita 2011). The detailed position of the clouds within the SNR blast wave cannot be reliably estimated from their projected distances from the boundary of the shell, but we will take this as being $\sim 25 \%$, as an upper limit, giving a mean shock age of $\lesssim 600 \mathrm{yr}$.

With the shock ram pressure given above, the mean pre-shock density at a given shock velocity is fully determined; for shock velocities of 300,350 and $400 \mathrm{~km} / \mathrm{s}$ the pre-shock particle densities are 160, 117 and $90 \mathrm{~cm}^{-3}$, respectively. None of these shocks can become fully radiative within this timescale. In the $600 \mathrm{yr}$ available, the $300 \mathrm{~km} / \mathrm{s}$ shock cools from $T_{e}=1.27 \times 10^{6} \mathrm{~K}$ to $T_{e}=$ $6.06 \times 10^{5} \mathrm{~K}$; the $350 \mathrm{~km} / \mathrm{s}$ shock from $T_{e}=1.72 \times 10^{6} \mathrm{~K}$ to $T_{e}=1.43 \times 10^{6} \mathrm{~K}$ and the $400 \mathrm{~km} / \mathrm{s}$ shock from $T_{e}=2.25 \times 10^{6} \mathrm{~K}$ to $T_{e}=2.07 \times 10^{6} \mathrm{~K}$.

The optical spectra of these shocks only contain emis- 


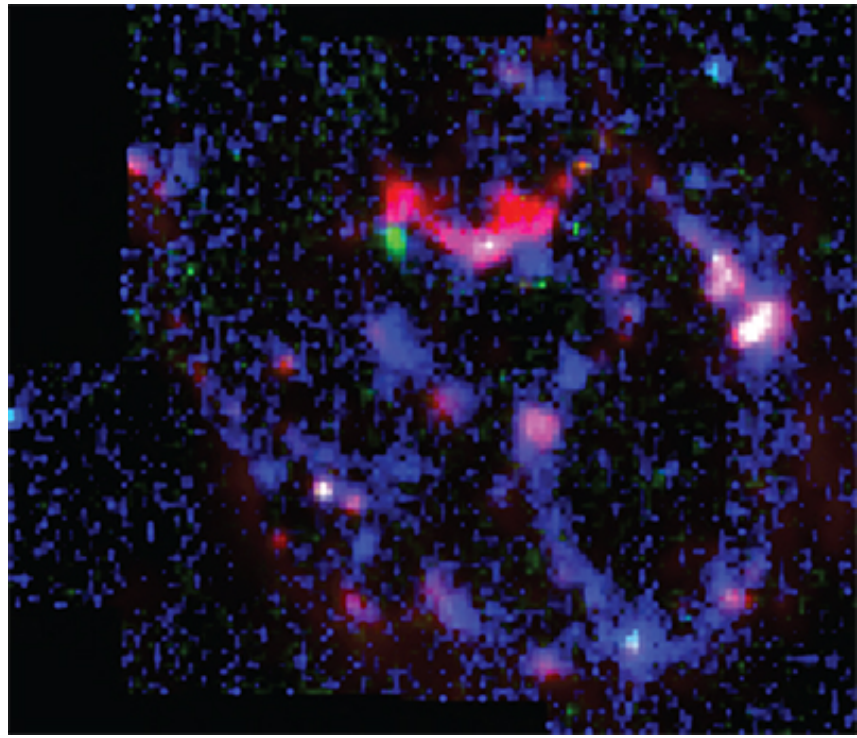

FIG. 13. - A WiFeS composite image in $\mathrm{H} \alpha$ (red), [Fe X] (green) and [Fe XIV] (blue). Note the greater spatial extent of the [Fe XIV] emission, and it close spatial correlation with the X-ray images of N132D. The green blob in this image is not real, but caused by leakage of high-velocity [O I] emission into the [Fe X] bandpass.

sion lines of hydrogen and helium, as well as lines of coronal species of S, Si, Ar, Fe and Ni. The $300 \mathrm{~km} / \mathrm{s}$ shock is too slow to reproduce the observed $[\mathrm{Fe} \mathrm{XIV}] /[\mathrm{Fe} \mathrm{X}]$ ratio, while the $400 \mathrm{~km} / \mathrm{s}$ overproduces [Fe XIV] relative to $[\mathrm{Fe} \mathrm{X}]$, and is somewhat too faint relative to the cloud shock and its precursor. The curves on Figure 12 are presented for the $350 \mathrm{~km} / \mathrm{s}$ shock. In this figure, we added the contribution of the fast shock to that of the cloud shock, assuming that the fast shock covers an area of either 3 times, or 10 times that of the cloud shock. We assume that dust has been fully destroyed by shattering and sputtering in these fast shocks. Adding this fast shock contribution, we now achieve a satisfactory fit with the observational diagnostics for both [Fe X] and [Fe XIV].

The WiFeS images of N132D support the hypothesis that the faster [Fe X] and [Fe XIV] - emitting shocks cover a greater area than the cloud shocks which are bright in $\mathrm{H} \alpha$. This is shown in Figure 13, in which we have constructed an image in $\mathrm{H} \alpha$ (red), [Fe X] (green) and [Fe XIV] (blue). In this image the clouds show up as red or mauve blobs, while the [Fe XIV] emission forms diffuse halos about these clouds, and is much more extensive. In general, the [Fe XIV] emission is much more closely spatially correlated with the Chandra X-Ray Observatory images (Borkowski et al. 2007), an effect which is also seen in both N49 in the LMC (Dopita et al. 2016) and in the SMC SNR; 1E 0102.2-7219 (Vogt et al. 2017).

\section{DETAILED CLOUD SHOCK MODELLING}

\subsection{Cloud Shock Parameters}

From the generalised shock diagnostic diagrams, we now turn to the derivation of the shock parameters of the individual shocked ISM clouds in N132D. First, using the shock velocities estimated from both the [O III] and $\mathrm{He} \mathrm{II} / \mathrm{He} \mathrm{I}$ diagnostic diagrams, the measured [S II] electron densities given by the [S II] $\lambda \lambda 6731 / 6717$ ratios, and the mean $[\mathrm{S} I \mathrm{II}]$ electron densities given from the model grid at the same shock velocity, we can estimate the pre-shock particle density, $n_{0}$, and the ram pressure driving the shock; $P_{\text {ram }}$. These are listed in Table 5. For the specific regions P08 and P09, the errors in the estimated shock velocities are great, as are the errors in $n_{0}$. The region P20 is an incompletely radiative shock so therefore the measured [S II] electron density represents an under-estimate of the true density in the [S II] recombination zone.

In the final column of Table 5 we give the time required for the post-shock plasma to cool to $1000 \mathrm{~K}, t_{1000 \mathrm{~K}}$ measured in years. At this point, the optical forbidden lines are no longer emitted in the plasma, and hydrogen is less than $2 \%$ ionised, so the shock is essentially fully radiative. We may conclude that these cloud shocks are somewhat older than $\left\langle t_{1000 \mathrm{~K}}\right\rangle=410 \mathrm{yr}$ (excluding P20).

The mean ram pressure for the clouds, $P_{\text {ram }}=3.1 \times$ $10^{-7}$ dynes $/ \mathrm{cm}^{2}$ is much higher than the mean pressure in the X-ray plasma. This may be estimated using the proton densities and electron temperatures listed in Williams et al. (2006). This gives $7.6 \times 10^{-8}$ dynes $\mathrm{cm}^{-2}$ in the NW region, and $5.3 \times 10^{-8}$ dynes $\mathrm{cm}^{-2}$ in the $\mathrm{S}$, for an average of $6.4 \times 10^{-8}$ dynes $\mathrm{cm}^{-2}$. Thus the ram pressure in the cloud shocks is typically $\sim 5$ times higher than in the surrounding X-ray plasma. The reason for this difference is discussed in Section 5 , below.

The mean pre-shock density inferred for these clouds is surprisingly high; $\left\langle n_{0}\right\rangle=243 \mathrm{~cm}^{-3}$ (excluding P08, P09 and P20). However, the pressure in the ISM of the LMC has been estimated using the excited C I emission along a number of sight lines by Welty et al. (2016). This analysis gave $\langle\log P / k\rangle=4.02 \mathrm{~cm}^{-3} \mathrm{~K}$. If the N132D clouds were characterised by the same ISM pressure before they were engulfed by the supernova shock wave (which is not at all a certain assumption), then their equilibrium temperature would have been $\sim 40 \mathrm{~K}$, and they can be therefore considered to be part of the cold neutral medium phase of the ISM.

\subsection{Chemical Abundances 85 Depletion Factors}

We selected the four brightest clouds; P12, P14, P15 and P18 for detailed study, with the aim of deriving more precise shock parameters, and to derive the chemical abundances of the elements in the ISM of the LMC through high-precision shock modelling.

In order to measure the goodness of fit of any particular model, we measure the degree to which it reproduces the density-sensitive [S II] $\lambda \lambda 6731 / 6717$ ratio, and we also seek to minimise the L1-norm for the fit. That is to say that we measure the modulus of the mean logarithmic difference in flux (relative to $\mathrm{H} \beta$ ) between the model and the observations viz.;

$$
\mathrm{L} 1=\frac{1}{m} \sum_{n=1}^{m}\left|\log \left[\frac{\left.F_{n} \text { (model }\right)}{F_{n}(\text { obs. })}\right]\right| .
$$

This procedure weights fainter lines equally with stronger lines, and is therefore more sensitive to the values of the input parameters. We simultaneously fit to those emission lines which are most sensitive to the controlling parameters of shock velocity and ram pressure; effectively He I, HeII, [O I], [O II] and [O III] as well as the $[\mathrm{NI}],[\mathrm{N} \mathrm{II}]$, and $[\mathrm{S} \mathrm{II}]$ lines. In addition, we estimate 
TABLE 5

THE PHYSICAL AND SHOCK PARAMETERS OF THE INTERSTELLAR CLOUDS IN N132D

\begin{tabular}{lccclll}
\hline \hline $\begin{array}{l}\text { Posn. } \\
\#\end{array}$ & $\begin{array}{c}v_{s} \\
\mathrm{~km} / \mathrm{s}\end{array}$ & $\begin{array}{c}{[\mathrm{S} \mathrm{II}]} \\
6731 / 6717\end{array}$ & $\begin{array}{l}n_{[\mathrm{SII}]} \\
\mathrm{cm}^{-3}\end{array}$ & $\begin{array}{l}n_{0} \\
\mathrm{~cm}^{-3}\end{array}$ & $\begin{array}{l}P_{\text {ram }} \\
\text { dynes} / \mathrm{cm}^{2}\end{array}$ & $\begin{array}{l}t_{1000 K} \\
\text { yr. }\end{array}$ \\
\hline & & & & & & \\
P01 & $225 \pm 40$ & 1.76 & 3760 & 304 & $3.5 \times 10^{-7}$ & 260 \\
P02 & $208 \pm 40$ & 1.83 & 4590 & 365 & $3.6 \times 10^{-7}$ & 165 \\
P03 & $225 \pm 50$ & 1.55 & 2180 & 176 & $2.0 \times 10^{-7}$ & 455 \\
P04 & $200 \pm 50$ & 1.87 & 5180 & 445 & $4.1 \times 10^{-7}$ & 222 \\
P05 & $263 \pm 35$ & 1.66 & 2880 & 198 & $3.2 \times 10^{-7}$ & 450 \\
P06 & $210 \pm 50$ & 1.76 & 3760 & 293 & $3.3 \times 10^{-7}$ & 185 \\
P07 & $238 \pm 70$ & 1.78 & 3840 & 323 & $4.0 \times 10^{-7}$ & 240 \\
P08 & $\sim 240$ & 1.70 & 3200 & $\sim 265$ & $\sim 3.4 \times 10^{-7}$ & $\sim 370$ \\
P09 & $\sim 240$ & 1.55 & 2180 & $\sim 180$ & $\sim 2.3 \times 10^{-7}$ & $\sim 550$ \\
P10 & $245 \pm 40$ & 1.68 & 3030 & 222 & $3.4 \times 10^{-7}$ & 395 \\
P11 & $285 \pm 35$ & 1.97 & 7250 & 426 & $5.3 \times 10^{-7}$ & 365 \\
P12 & $235 \pm 25$ & 1.74 & 3560 & 308 & $3.6 \times 10^{-7}$ & 290 \\
P13 & $250 \pm 40$ & 1.45 & 1690 & 129 & $1.8 \times 10^{-7}$ & 750 \\
P14 & $228 \pm 30$ & 1.53 & 2070 & 170 & $2.0 \times 10^{-7}$ & 450 \\
P15 & $205 \pm 25$ & 1.43 & 1610 & 132 & $1.4 \times 10^{-7}$ & 430 \\
P16 & $238 \pm 50$ & 1.78 & 3970 & 288 & $3.9 \times 10^{-7}$ & 275 \\
P17 & $210 \pm 40$ & 1.45 & 1690 & 132 & $1.5 \times 10^{-7}$ & 410 \\
P18 & $360 \pm 20$ & 1.52 & 2020 & 117 & $3.7 \times 10^{-7}$ & 1100 \\
P19 & $310 \pm 40$ & 1.53 & 2070 & 131 & $3.1 \times 10^{-7}$ & 820 \\
P20 & $190 \pm 20$ & 1.16 & 760 & 72 & $>5.4 \times 10^{-8}$ & $\cdots$ \\
& & & & & & \\
\hline Av: & 246 & & & 243 & $3.1 \times 10^{-7}$ & 410 \\
\hline & & & & & & \\
\hline
\end{tabular}

the gas-phase heavy element abundances using the bright

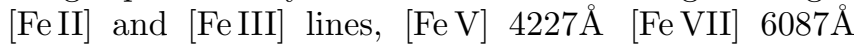

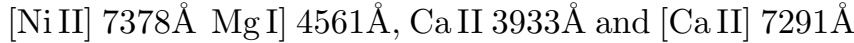
lines. We assume that the more excited species of Fe are depleted in the gas phase by the same amount as Fe III. For the reason given above we do not attempt to fit either the $[\mathrm{Fe} \mathrm{X}] 6374 \AA$ or the [Fe XIV] $5303 \AA$ lines, given that they arise in a separate phase of the ISM. Other elements such as $\mathrm{Ne}, \mathrm{Ar}$ and $\mathrm{Cl}$ are fit using the [Ne III] $3969 \AA$ [Ar III $] 7136 \AA$ and $[\mathrm{Cl}$ II] $8579 \AA$ lines.

Our models simultaneously optimise the fit to 40 emission lines, and we manually iterate shock velocity, ram pressure and chemical abundances until the fit is optimised. The results for these four clouds are shown in Table 6. The L1-norm provides a good estimate for the error on the abundance of those elements fitted with only one or two emission lines. For $\mathrm{O}, \mathrm{N}$ and $\mathrm{S}$ the accuracy is higher. In Table 2, we have adopted the abundances of $\mathrm{Mg}, \mathrm{Ca}, \mathrm{Fe}$ and Ni from Russell \& Dopita (1992) in order to estimate the depletion factors of these elements.

The best fit was achieved without the inclusion of the precursor emission, which in principle could account for as much as $25 \%$ of the total emission. There are two possible reasons for this. First, the finite extent of the cloud may limit the length of the precursor, which is maximised in our plane-parallel models. Second, the local curvature of the shock front may allow significant escape of the upstream ionising UV photons. This is suggested by the fact that our models of these four clouds systematically overestimate the He II $\lambda 4686 / \mathrm{He}$ I $\lambda 5876$ ratio, which suggests that the incoming plasma is over-ionised by the computed precursor radiation field given by the model.

The derived depletion factors for $\mathrm{Mg}$ given in Table 6 are likely to have very large, and unquantifiable errors
TABLE 6

GOODNESS OF FIT, SHOCK PARAMETERS, IONIC DEPLETIONS AND CHEMICAL ABUNDANCES, $12+\log ([\mathrm{X} / \mathrm{H}])$, FOR ClOUdS IN N132D.

\begin{tabular}{lrrrr}
\hline \hline Posn: & P12 & P14 & P15 & P18 \\
\hline & & & & \\
L1-norm & 0.059 & 0.083 & 0.086 & 0.081 \\
$v_{s}(\mathrm{~km} / \mathrm{s})$ & $210 \pm 25$ & $225 \pm 50$ & $210 \pm 30$ & $260 \pm 30$ \\
$n(\mathrm{H})_{0}\left(\mathrm{~cm}^{-3}\right)$ & $410 \pm 30$ & $325 \pm 25$ & $160 \pm 25$ & $216 \pm 25$ \\
$P_{\text {ram }}$ & 3.88 & 3.57 & 1.51 & 2.90 \\
$\left(\times 10^{7}\right.$ dynes $\left.\mathrm{cm}^{2}\right)$ & & & & \\
& & & & \\
\hline $\log D_{\text {FeII }}$ & -0.13 & -0.13 & -0.13 & -0.13 \\
$\log D_{\text {FeIII }}$ & -0.67 & -0.63 & -0.58 & -0.47 \\
$\log D_{\text {MgII }}$ & -0.94 & -0.57 & -1.33 & -0.74 \\
$\log D_{\text {CaII }}$ & -0.30 & -0.05 & -0.06 & -0.04 \\
$\log D_{\text {NiII }}$ & -0.69 & -0.28 & -0.30 & -0.40 \\
\hline & & & & \\
$\mathrm{He}$ & 10.84 & 10.86 & 10.85 & 10.86 \\
$\mathrm{~N}$ & 7.11 & 7.08 & 7.15 & 7.15 \\
$\mathrm{O}$ & 8.28 & 8.30 & 8.32 & 8.32 \\
$\mathrm{Ne}$ & 7.45 & 7.42 & 7.45 & 7.45 \\
$\mathrm{Mg}$ & 7.47 & 7.47 & 7.47 & 7.47 \\
$\mathrm{~S}$ & 6.95 & 7.10 & 7.00 & 7.00 \\
$\mathrm{Cl}$ & 4.35 & 4.90 & 4.35 & 5.03 \\
$\mathrm{Ar}$ & 6.13 & 5.65 & 5.68 & 5.68 \\
$\mathrm{Ca}$ & 5.97 & 5.97 & 5.97 & 5.97 \\
$\mathrm{Fe}$ & 7.23 & 7.23 & 7.23 & 7.23 \\
$\mathrm{Ni}$ & 5.96 & 5.96 & 5.96 & 5.96 \\
\hline \hline & & & & \\
\hline
\end{tabular}

attached to them. There is only one $\mathrm{Mg}$ line detected, and it is produced by neutral $\mathrm{Mg}$ in the tail of the recombination zone of the shock. Given the low ionisation potential of this species $(7.64 \mathrm{eV})$, the $\mathrm{MgI}$ ] line strength will be highly dependent on the unknown stellar UV radiation field, which by ionising this species would depress the line strength and lead to a larger depletion factor being inferred.

For the remaining elements we derive the following mean LMC interstellar abundances, $12+\log ([\mathrm{X} / \mathrm{H}])$, of He: $10.86 \pm 0.05, \mathrm{~N}: 7.12 \pm 0.07, \mathrm{O}: 8.31 \pm 0.04$, Ne: $7.44 \pm 0.08, \mathrm{~S}: 7.01 \pm 0.06, \mathrm{Cl}: 4.66 \pm 0.11$ and Ar: $5.78 \pm 0.11$. These abundances are generally similar to those obtained in our detailed shock fitting of the supernova remnant N49 (Dopita et al. 2016); He: 10.92, N: 7.10, O: 8.46, Ne: 7.79, S: 7.05, Cl: 5.2 and Ar: 6.1 .

These abundances should also be compared with those from Russell \& Dopita (1992): He: $10.94 \pm 0.03, \mathrm{~N}$ : $7.14 \pm 0.15, \mathrm{O}: 8.35 \pm 0.06$, Ne: $7.61 \pm 0.05$, S: $6.70 \pm 0.09$, $\mathrm{Cl}: 4.76 \pm 0.08$ and Ar: $6.29 \pm 0.25$. Given the differences in the methodology used to derive these, and the changes in key atomic input parameters over the years, the agreement between these two is as good as could be expected. No systematic difference is seen for the elements most important in the modelling; $\mathrm{N}$ and $\mathrm{O}$, but the derived $\mathrm{He}, \mathrm{Ne}, \mathrm{S}, \mathrm{Cl}$ and $\mathrm{Ar}$ abundances are only marginally consistent with each other. What is now required is a rederivation of the abundances using H II regions and the same modelling code to compare with the SNR-derived abundances.

The derived abundances can also be compared to more recent determinations from stellar observations. Korn et al. (2005) gave an new analysis of a slow-rotating B star in NGC 2002 which gave N: $6.99 \pm 0.2$, O: $8.29 \pm 0.2$, and $\mathrm{Mg}: 7.44 \pm 0.2$. These values differed very little from 
his earlier work (Korn et al. 2002).

With respect to the refractory elements, Korn et al. (2000) derived appreciably lower $\mathrm{Mg}$ and $\mathrm{Fe}$ abundances than Russell \& Dopita (1992); Mg: $6.96 \pm 0.22$ and Fe: $7.09 \pm 0.15$. Using these values, our results are consistent with full destruction of the Fe-bearing grains in the [Fe II]-emitting zone, and the estimated $\mathrm{Mg}$ depletion factor is lowered to only $\log D_{\mathrm{MgII}}=-0.38$. However, the later Korn et al. (2002) paper gave Mg: $7.37 \pm 0.06$ and Fe: $7.33 \pm 0.0 .03$, which is in much closer agreement with the Russell \& Dopita (1992) estimates.

In conclusion, we can be fairly confident of the LMC $\mathrm{He}, \mathrm{N}$ and $\mathrm{O}$ abundances; He: $10.92 \pm 0.04, \mathrm{~N}: 7.12 \pm$ 0.09 and $\mathrm{O}: 8.32 \pm 0.06$. For the other non-refractory elements the recommended values are Ne: $7.52 \pm 0.09$, S: $7.00 \pm 0.15, \mathrm{Cl}: 4.70 \pm 0.08$ and Ar: $5.78 \pm 0.25$.

Our results are consistent with nearly full destruction of the $\mathrm{Fe}$-containing grains in these $210-260 \mathrm{~km} / \mathrm{s}$ shocks, but the $\mathrm{Mg}$-containing grains appear to be only partially destroyed. This argues for a different carrier for the two metals, possibly iron(II) oxide $(\mathrm{FeO})$ for iron and the magnesium silicates, forsterite $\left(\mathrm{Mg}_{2} \mathrm{SiO}_{4}\right)$ and enstatite $\left(\mathrm{MgSiO}_{3}\right)$ in the case of $\mathrm{Mg}$.

The destruction of $\mathrm{Fe}-$ containing grains appears to have proceeded to a greater extent in N132D than in N49 (Dopita et al. 2016). Although the estimated shock velocities are similar $(210-260 \mathrm{~km} / \mathrm{s}$ in N132D and 200 $250 \mathrm{~km} / \mathrm{s}$ in N49), the higher ram pressures and higher pre-shock densities in N132D $\left(160-410 \mathrm{~cm}^{-3}\right)$ compared with N49 $\left(\sim 80 \mathrm{~cm}^{-3}\right)$ has facilitated this greater fractional dust destruction.

In both N49 and in N132D, the grain destruction in the faster, partially-radiative shocks producing [Fe X] and [Fe XIV] emission is much more advanced. In both these SNR, the shock velocity in these highly-ionised zones is $\sim 350 \mathrm{~km} / \mathrm{s}$. Thus, thermal sputtering and non-thermal sputtering due to the high relative velocity of the dust grains relative to the hot-post shock plasma (Barlow 1978; McKee et al. 1987; Seab 1987; Jones et al. 1994) dominates the grain destruction in these fast shocks, while gyro-acceleration (aka betatron acceleration) caused by the compression of the magnetic field in the cooling zone of the shock resulting in grain shattering and vaporisation (Spitzer 1976, Borkowski \& Dwek 1995) appears to dominate as the grain destruction process in the slower, denser shocks.

\subsection{The partially-radiative region P20}

We already noted in Section 3 that region P20 has anomalously strong [O III] emission - see Figure 7 . Furthermore, the [S II] lines indicate a low density compared with the other regions which produces the low value of the ram pressure given in Table 5. All of these point to a shock which has not yet become fully radiative. In this case, it appears that the photoionised precursor is brighter in the optical lines than the shock itself. The extremely strong [O III] would arise in this precursor.

The observed spectrum of this region proved very difficult to model. Our "best" model has a relatively poor fit, with an L1-norm of 0.21 . This model has a $\sim 400 \mathrm{yr}$ old shock with $v_{s}=300 \mathrm{~km} / \mathrm{s}$ moving into a medium with a pre-shock $\mathrm{H}$ density $n_{0}=235 \mathrm{~cm}^{-3}$. In this shock the plasma cools from an initial temper-

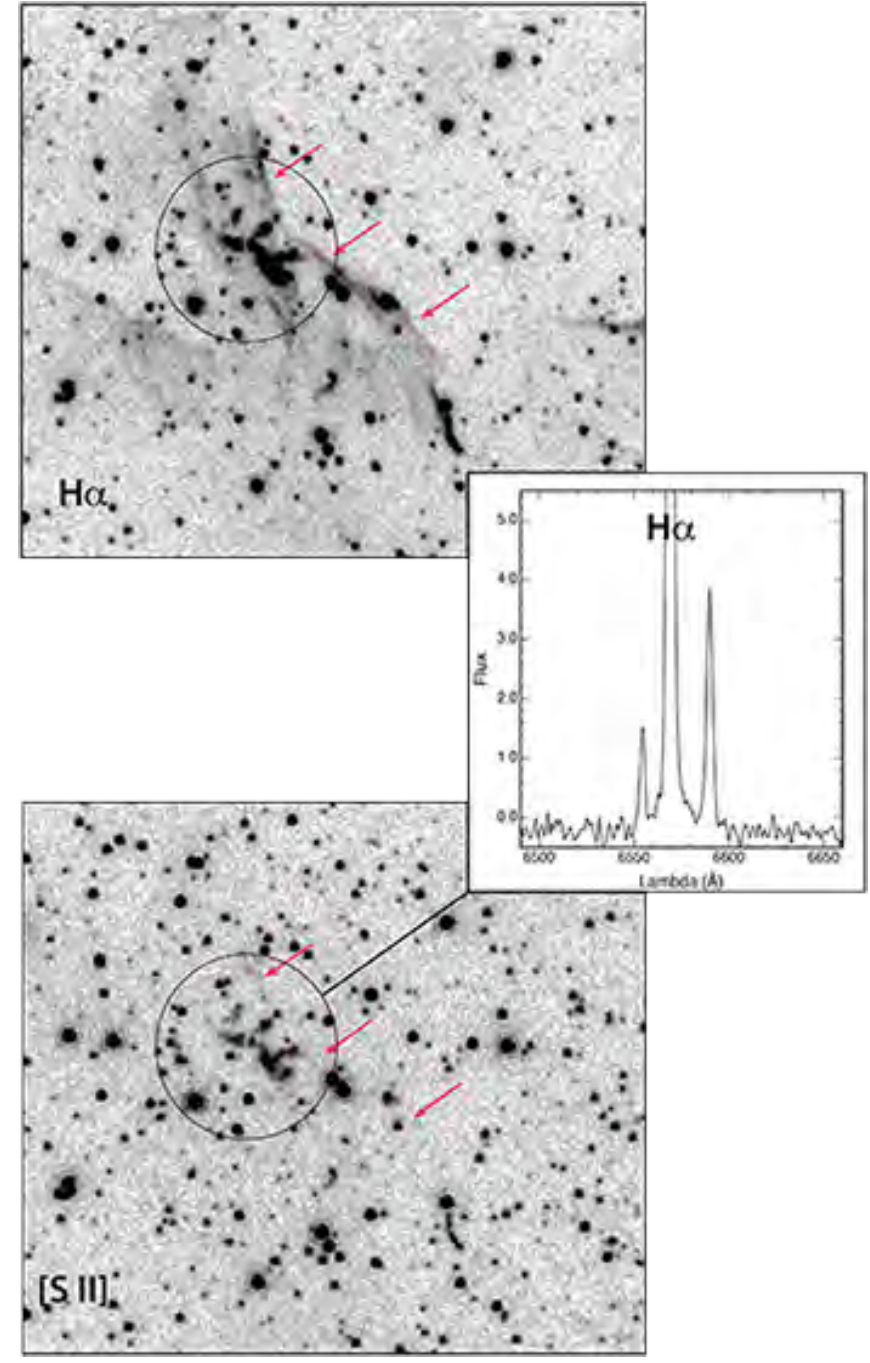

Fig. 14. - The region P08 as seen by HST in H $\alpha$ (top panel) and in [S II] (lower panel). The WiFeS extraction aperture is shown as a black circle, and the resultant spectrum around $\mathrm{H} \alpha$ is shown in the inset. The Flux units are the same as in Figure 4 Note the broad wings of $\mathrm{H} \alpha$ extending out to $\pm 900 \mathrm{~km} / \mathrm{s}$. This broad emission is presumably originating from the filaments which are marked with red arrows on the images.

ature of $T_{e}=1.2 \times 10^{6} \mathrm{~K}$ to a final temperature of $T_{e}=8.2 \times 10^{5} \mathrm{~K}$. The precursor material is assumed to have no dust destruction with $\log D_{F e}=-1.00$, and the density of the pre-shock gas $n_{0}=235 \mathrm{~cm}^{-3}$, and is illuminated by the UV radiation field of the partially-radiative shock in plane-parallel one-sided geometry. This gives a precursor thickness of $\sim 0.12 \mathrm{pc}$ (at the point where $\mathrm{H}$ is $50 \%$ ionised). For this configuration, the shock produces only $4.5 \%$ of the total $\mathrm{H} \beta$ luminosity. Table 7 provides comparison of the key emission lines predicted by the model, compared with the observations.

Note the extreme discrepancy in the predicted strength of the [O III] $\lambda 4363$ line. From the model, the predicted temperature in the $\mathrm{O}$ III zone is $T_{e}=13600 \mathrm{~K}$, while the data suggest an electron temperature of $T_{e} \sim 49000 \mathrm{~K}$ in this zone. Similar, but smaller, temperature discrepancies are suggested for the $\mathrm{O}$ II and N II zones. This requires another form of heating in the precursor such as electron conduction, cosmic ray heating or else ioni- 
TABLE 7

THE PARTIALLY-RADIATIVE SHOCK MODEL FIT TO THE OBSERVATIONS OF P20 $(\mathrm{H} \beta=100)$.

\begin{tabular}{lccc}
\hline \hline$\lambda(\AA)$ & ID & Obs. & Model \\
& & & \\
\hline 3727,9 & {$[\mathrm{O} \mathrm{II}]$} & 419 & 555 \\
3867 & {$[\mathrm{Ne} \mathrm{III}]$} & 82 & 62 \\
3835 & $\mathrm{H} \eta$ & 6 & 7 \\
3967 & {$[\mathrm{Ne} \mathrm{III}]$} & 25 & 19 \\
3970 & $\mathrm{H} \epsilon$ & 15 & 15 \\
4969,76 & {$[\mathrm{~S} \mathrm{II}]$} & 9 & 8 \\
4340 & $\mathrm{H} \gamma$ & 45 & 46 \\
4363 & {$[\mathrm{O} \mathrm{III}]$} & 84 & 7 \\
4696 & $\mathrm{He} \mathrm{II}$ & 14 & 35 \\
4861 & $\mathrm{H} \beta$ & 100 & 100 \\
4959 & {$[\mathrm{O} \mathrm{III}]$} & 326 & 165 \\
5007 & {$[\mathrm{O} \mathrm{III}]$} & 967 & 476 \\
5755 & {$[\mathrm{~N} \mathrm{II}]$} & 8 & 2 \\
5876 & $\mathrm{He} \mathrm{I}$ & 6 & 6 \\
6548 & {$[\mathrm{~N} \mathrm{II}]$} & 33 & 21 \\
6563 & $\mathrm{H} \alpha$ & 294 & 318 \\
6584 & {$[\mathrm{~N} \mathrm{II}]$} & 95 & 63 \\
6717 & {$[\mathrm{~S} \mathrm{II}]$} & 121 & 83 \\
6731 & {$[\mathrm{~S} \mathrm{II}]$} & 142 & 62 \\
7136 & {$[\mathrm{Ar} \mathrm{III}]$} & 17 & 8 \\
7319 & {$[\mathrm{O} \mathrm{II}]$} & 46 & 10 \\
7329 & {$[\mathrm{O} \mathrm{II}]$} & 25 & 8 \\
\hline \hline
\end{tabular}

sation by the general EUV radiation field of the nebula. Apart from these temperature discrepancies, the [S II] $\lambda \lambda 6731 / 6717$ ratio also requires a higher electron density; $n_{[\mathrm{SII}]} \sim 760 \mathrm{~cm}^{-3}$.

\subsection{The Balmer-Dominated Shocks in P08}

We noted above that the region P08 also displays an anomalous spectrum, deficient in the forbidden lines with respect to the Balmer lines, with the [O III] lines being particularly weak - see Figure 7. The cause of this appears to be "non-radiative" or Balmer-dominated shocks. These occur when a fast shock runs into a cold, essentially un-ionised ISM. A narrow component of the Balmer lines arises from direct collisional excitation of Hydrogen by the fast electrons, and a broad Balmer component is produced by charge exchange with fast protons behind the shock and subsequent collisional excitation of the fast neutral hydrogen resulting by the hot electrons (Chevalier \& Raymond 1978; Chevalier et al. 1980) - see the reviews by Heng (2010) and Ghavamian et al. (2013), and references therein.

In Figure 14, we show the HST view of the P08 region, and the spectrum extracted from the WiFeS data cube. It is most likely that the broad component of $\mathrm{H} \alpha$ seen in the spectroscopy arises from the filaments which are visible in the $\mathrm{H} \alpha$ image, but not in the $[\mathrm{S} \mathrm{II}]$ image. A broad component is also weakly detected in $\mathrm{H} \beta$. This would add N132D to the select group of SNR in which this has been detected - which includes Tycho, SN 1006, RCW 86, the Cygnus Loop and N103B and SNR 0519-69.0 (DEM N71) in the LMC (Tuohy et al. 1982; Ghavamian et al. 2001, 2002, 2017). Most, but not all, of these remnants are from 'Type la supernova explosions, since these do not produce a burst of strong EUV radiation to pre-ionise the pre-shock medium. However, if the ISM is shielded from the EUV flash in some way, or if it is dense enough to recombine in the period between the explosion and the arrival of the shock, then nothing precludes the formation of a Balmer-dominated shock. Given the inferred age of the SNR, the recombination condition would imply that the shock is passing through a medium with a density $\gtrsim 20 \mathrm{~cm}^{-3}$. The width of the $\mathrm{H} \alpha$ line suggests that the shock velocity in the Balmer-dominated shock is of order $\sim 900 \mathrm{~km} / \mathrm{s}$. This is similar to the blast wave velocity derived from the X-ray data (Favata et al. 1997. Hughes et al. 1998; Borkowski et al. 2007), so it seems extremely likely that the broad Balmer lines arise in the blast wave itself.

\section{CONCLUSIONS}

The environment of N132D provides an excellent sampling of shocked clouds in the Bar of the LMC. The typical physical size of these clouds is $\sim 1.0 \mathrm{pc}$. Given that they have a typical pre-shock density of $\sim 240 \mathrm{~cm}^{-3}$ (from Table 5), we can infer cloud masses of few solar masses. Using the images from Figure 3 and the preshock densities from Table 5. we obtain masses in the range $0.1-20 \mathrm{M}_{\odot}$ with a mean of $\sim 4 \mathrm{M}_{\odot}$. Thus we infer these clouds initially represented typical ISM selfgravitating Bonnor-Ebert spheres such as those recently investigated on a theoretical basis by Sipilä et al. (2011), Fischera (2014) and Sipilä et al. (2017). Given that the shock which moves into them following the passage of the supernova blast-wave is strongly compressive, and that such Bonnor-Ebert spheres can be marginally stable against collapse, it is tempting to imagine that the supernova shock may later induce formation of $\sim 1.0 M_{\odot}$ stars within the cores of those clouds. 
Now, let us consider the discrepancy between the thermal pressure measured by the X-rays, and the ram pressure driving the cloud shocks, pointed out in Section 4.1. The X-ray plasma has a thermal pressure of $P_{\text {therm }}=6.4 \times 10^{-8}$ dynes $\mathrm{cm}^{-2}$, while the ram pressure in the clouds is $P_{\text {ram }}=3.1 \times 10^{-7}$ dynes $\mathrm{cm}^{-2}$. However, the pressure driving the cloud shocks is provided by the stagnation pressure behind the cloud bow shock or bow wave produced in the expanding thermal plasma which fills the SNR. This is always greater than the pressure in the pre-shock hot thermal plasma. The geometry of this interaction is pictured in McKee \& Cowie (1975); Hester \& Cox (1986) and Farage et al. (2010). In the framework of a plane-parallel strong shock engulfing the cloud the stagnation pressure is about twice as large as the thermal pressure (McKee \& Cowie 1975, Hester \& Cox 1986) and this is only very weakly dependent to the Mach number of the primary blast wave (see Hester \& Cox (1986), Table 2).

The X-ray data enable us to estimate the stagnation pressure in two ways. First, we may use the Sedov theory to estimate the blast wave velocity. Using the figures given in Hughes et al. (1998) for the explosion energy, age and pre-shock density in the SNR, we derive the blast-wave velocity, $v_{\mathrm{B}}=830 \mathrm{~km} / \mathrm{s}$. Alternatively, we can use the measured thermal plasma temperatures to derive the blast wave velocity, using the relation $T_{\mathrm{B}}=\left(3 \mu m_{H} / 16 k\right) v_{\mathrm{B}}^{2}$, where $m_{H}$ is the pre-shock hydrogen density and the molecular weight $\mu$ is appropriate to a fully-ionised plasma. Estimates of the thermal temperature are in the range $0.68<k T<0.8 \mathrm{keV}$ (Favata et al. 1997, Hughes et al. 1998: Borkowski et al. 2007), which implies a blast wave velocity of $\sim 760 \mathrm{~km} / \mathrm{s}$. Therefore, the ram pressure associated with expansion is $\sim 4 \times 10^{-8}$ dynes $\mathrm{cm}^{-2}$. Adding this to the thermal pressure gives an estimate of the stagnation pressure, $\sim 1.0 \times 10^{-7}$ dynes $\mathrm{cm}^{-2}$.

The remaining difference between the ram pressure of the cloud shocks and the estimated stagnation pressure is a factor of three. This difference may be accounted for by the fact that the cloud shocks are convergent towards the centre of mass, a possibility raised in the context of the Cygnus Loop SNR by Hester \& Cox (1986) as a promising mechanism for increasing pressure in shocked dense clouds. For shocks in self-gravitating isothermal spheres such as those considered here, families of self-similar solutions have been obtained by Lou \& Shi (2014). In such shocks, the energy density increases as the shock moves toward the center of the cloud, increasing the ram pressure. This effect can easily account for the difference between the measured ram pressure of the cloud shocks and the estimated stagnation pressure. Additionally, convergent cloud shocks are unstable in the presence of small deviations from sphericity (Kimura \& Tosa 1990), and elongated clouds may break up into separate globules (Kimura \& Tosa 1991). Such instabilities are the likely reason for the complex morphologies of the shocks in individual clouds seen in Figure 3 .

Because the shocked clouds of N132D represent gravitationally-confined samples of the ISM as they existed before the supernova event, and because these clouds are dense enough that they would not be appreciably affected in their chemical composition by any
TABLE 8

RECOMMENDED LMC CHEMICAL ABUNDANCES.

\begin{tabular}{lc}
\hline \hline Element & $12+\log ([\mathrm{X} / \mathrm{H}])$ \\
\hline & \\
$\mathrm{He}$ & $10.92 \pm 0.04$ \\
$\mathrm{C}$ & $8.06 \pm 0.09$ \\
$\mathrm{~N}$ & $7.12 \pm 0.09$ \\
$\mathrm{O}$ & $8.32 \pm 0.06$ \\
$\mathrm{Ne}$ & $7.52 \pm 0.09$ \\
$\mathrm{Mg}$ & $7.37 \pm 0.06$ \\
$\mathrm{Si}$ & $7.10 \pm 0.07$ \\
$\mathrm{~S}$ & $7.00 \pm 0.15$ \\
$\mathrm{Cl}$ & $4.70 \pm 0.08$ \\
$\mathrm{Ar}$ & $5.78 \pm 0.25$ \\
$\mathrm{Fe}$ & $7.33 \pm 0.03$ \\
\hline \hline
\end{tabular}

pre-supernova mass-loss, they present ideal samples of pristine ISM in the LMC. Our radiative shock analysis has enabled us to estimate accurate gas-phase chemical abundances for a number of elements.

From our model grid, we have demonstrated that the [O III] $\lambda \lambda 4363 / 5007$ ratio is a good indicator of shock velocity, and that the He II/He I $\lambda \lambda 4686 / 5876$ ratio may also be used for this purpose, although it is rather less reliable. Typical shock velocities are $\sim 240 \mathrm{~km} / \mathrm{s}$, in agreement with the shock velocities inferred from the kinematics and the measured emission line widths. Using these emission line diagnostics, we have analysed the depletion onto dust in the various ionic stages of $\mathrm{Fe}$, and in $\mathrm{Mg}$ II $\mathrm{Ca}$ II and Ni II. In common with the SNR N49, we find that dust has been mostly destroyed in the region emitting the [Fe II] lines, while a smaller fraction has been destroyed in the Fe III and Fe VII zones, consistent with the grain destruction models of Seab \& Shull (1983) and Borkowski \& Dwek (1995). However, Mg II shows an appreciably higher depletion factor, suggesting that the $\mathrm{Mg}$ silicates are more resistant to destruction than the Fe-bearing grains.

It is clear that the highly ionised species of iron; Fe X and Fe XIV originate in faster, partially radiative, filamentary and spatially extensive shocks surrounding the dense clouds. Some of these shocks may well be part of the primary blast wave of the SNR. In these, most of the refractory elements have been destroyed by thermal sputtering.

A detailed shock analysis of the four brightest clouds has allowed us to determine the chemical abundances of a number of elements. Comparing these with values given earlier, and with stellar abundance determinations we can now provide a "recommended" set of LMC abundances, which we present here in Table 8 . Here, for completeness, the abundances of $\mathrm{C}, \mathrm{Mg}, \mathrm{Si}$ and $\mathrm{Fe}$ have been taken from the work of Korn et al. (2002) using Magellanic Cloud B-stars in NGC2004.

Finally we have identified two anomalous regions, P08 and P20. The former contains a contribution from fast Balmer-dominated shocks, while the latter represents an unusual partially-radiative shock, dominated by precursor emission which seems to be heated by an unknown source to very high electron temperatures.

\section{ACKNOWLEDGMENTS}


MD and RS acknowledge the support of the Australian Research Council (ARC) through Discovery project DP16010363. Parts of this research were conducted by the Australian Research Council Centre of Excellence for All Sky Astrophysics in 3 Dimensions (ASTRO 3D), through project number CE170100013. FPAV and IRS thank the CAASTRO AI travel grant for generous support. IRS was supported by the ARC through the Future Fellowship grant FT1601000028. AJR is supported by the Australian Research Council through Future Fellowship grant FT170100243.

This research has made use of MATPLOTLIB (Hunter 2007), ASTROPY, a community-developed core PYTHON package for Astronomy Astropy Collaboration et al. 2013), APLPY, an open-source plotting package for PYTHON(Robitaille \& Bressert 2012), and MONTAGE, funded by the National Science Foundation under Grant Number ACI-1440620 and previously funded by the National Aeronautics and Space Administration's Earth Science Technology Office, Computation Technologies Project, under Cooperative Agreement Number NCC5626 between NASA and the California Institute of Technology.

This research has also made use of DRIZZLEPAC, a product of the Space Telescope Science Institute, which is operated by AURA for NASA, of the ALADIN interactive sky atlas (Bonnarel et al. 2000), of SAOIMAGE DS9
(Joye \& Mandel 2003) developed by Smithsonian Astrophysical Observatory, of NASA's Astrophysics Data System, and of the NASA/IPAC Extragalactic Database Helou et al. (1991) which is operated by the Jet Propulsion Laboratory, California Institute of Technology, under contract with the National Aeronautics and Space Administration. Support for MAST for non-HST data is provided by the NASA Office of Space Science via grant NNX09AF08G and by other grants and contracts. This research has also made use of NASA's Astrophysics Data System.

This work has made use of data from the European Space Agency (ESA) mission Gaia (https://www.cosmos.esa.int/gaia), processed by the Gaia Data Processing and Analysis Consortium (DPAC, https://www.cosmos.esa.int/web/gaia/ dpac/consortium). Funding for the DPAC has been provided by national institutions, in particular the institutions participating in the Gaia Multilateral Agreement. Some of the data presented in this paper were obtained from the Mikulski Archive for Space Telescopes (MAST). STScI is operated by the Association of Universities for Research in Astronomy, Inc., under NASA contract NAS5-26555. Support for MAST for non-HST data is provided by the NASA Office of Space Science via grant NNX09AF08G and by other grants and contracts.

\section{REFERENCES}

Astropy Collaboration, Robitaille, T. P., Tollerud, E. J., et al. 2013, A\&A, 558, A33

Baldwin, J. A., Phillips, M. M., \& Terlevich, R. 1981, PASP, 93, 5 Barlow, M. J. 1978, MNRAS, 183, 367

Blair, W. P., Morse, J. A., Raymond, J. C., et al. 2000, ApJ, 537, 667

Bonnarel, F., Fernique, P., Bienaymé, O., et al. 2000, A\&AS, 143 33

Borkowski, K. J., \& Dwek, E. 1995, ApJ, 454, 254

Borkowski, K. J., Hendrick, S. P., \& Reynolds, S. P. 2007, ApJ, $671, \mathrm{~L} 45$

Chevalier, R. A., Kirshner, R. P., \& Raymond, J. C. 1980, ApJ, 235,186

Chevalier, R. A., \& Raymond, J. C. 1978, ApJ, 225, L27

Childress, M., Vogt, F., Nielsen, J., \& Sharp, R. 2014a, PyWiFeS: Wide Field Spectrograph data reduction pipeline, Astrophysics Source Code Library, , , ascl:1402.034

Childress, M. J., Vogt, F. P. A., Nielsen, J., \& Sharp, R. G. 2014b, Ap\&SS, 349, 617

Danziger, I. J., \& Dennefeld, M. 1976, ApJ, 207, 394

Dopita, M., Hart, J., McGregor, P., et al. 2007, Ap\&SS, 310, 255

Dopita, M., Rhee, J., Farage, C., et al. 2010, Ap\&SS, 327, 245

Dopita, M. A., Payne, J. L., Filipović, M. D., \& Pannuti, T. G. 2012, MNRAS, 427, 956

Dopita, M. A., Seitenzahl, I. R., Sutherland, R. S., et al. 2016 ApJ, 826, 150

Dopita, M. A., \& Sutherland, R. S. 2017, ApJS, 229, 35

Farage, C. L., McGregor, P. J., Dopita, M. A., \& Bicknell, G. V. 2010, ApJ, 724, 267

Favata, F., Vink, J., Parmar, A. N., Kaastra, J. S., \& Mineo, T. 1997, A\&A, 324, L45

Feast, M. W. 1964, MNRAS, 127, 195

Fischera, J. 2014, A\&A, 571, A95

Fitzpatrick, E. L. 1986, AJ, 92, 1068

Gaia Collaboration, Prusti, T., de Bruijne, J. H. J., et al. 2016a, A\&A, 595, A1

Gaia Collaboration, Brown, A. G. A., Vallenari, A., et al. 2016b, A\&A, 595, A2

Ghavamian, P., Raymond, J., Smith, R. C., \& Hartigan, P. 2001, ApJ, 547, 995

Ghavamian, P., Schwartz, S. J., Mitchell, J., Masters, A., \& Laming, J. M. 2013, Space Sci. Rev., 178, 633

Ghavamian, P., Seitenzahl, I. R., Vogt, F. P. A., et al. 2017, ApJ, 847,122

Ghavamian, P., Winkler, P. F., Raymond, J. C., \& Long, K. S. 2002, ApJ, 572, 888
Helou, G., Madore, B. F., Schmitz, M., et al. 1991, in Astrophysics and Space Science Library, Vol. 171, Databases and On-line Data in Astronomy, ed. M. A. Albrecht \& D. Egret, 89-106

Heng, K. 2010, PASA, 27, 23

Hester, J. J., \& Cox, D. P. 1986, ApJ, 300, 675

Hughes, J. P., Hayashi, I., \& Koyama, K. 1998, ApJ, 505, 732

Hunter, J. D. 2007, Computing in Science and Engineering, 9, 90

Hwang, U., Hughes, J. P., Canizares, C. R., \& Markert, T. H. 1993, ApJ, 414, 219

Jenkins, E. B. 2009, ApJ, 700, 1299

Jones, A. P., Tielens, A. G. G. M., Hollenbach, D. J., \& McKee, C. F. 1994, ApJ, 433, 797

Joye, W. A., \& Mandel, E. 2003, in Astronomical Society of the Pacific Conference Series, Vol. 295, Astronomical Data Analysis Software and Systems XII, ed. H. E. Payne, R. I. Jedrzejewski, \& R. N. Hook, 489

Kimura, T., \& Tosa, M. 1990, MNRAS, 245, 365

-. 1991, MNRAS, 251, 664

Korn, A. J., Becker, S. R., Gummersbach, C. A., \& Wolf, B. 2000, A\&A, 353, 655

Korn, A. J., Keller, S. C., Kaufer, A., et al. 2002, A\&A, 385, 143

Korn, A. J., Nieva, M. F., Daflon, S., \& Cunha, K. 2005, ApJ, 633,899

Lasker, B. M. 1980, ApJ, 237, 765

Lou, Y.-Q., \& Shi, C.-H. 2014, MNRAS, 442, 741

McKee, C. F., \& Cowie, L. L. 1975, Ap.J, 195, 715

McKee, C. F., Hollenbach, D. J., Seab, G. C., \& Tielens, A. G. G. M. 1987, ApJ, 318, 674

Nicholls, D. C., Sutherland, R. S., Dopita, M. A., Kewley, L. J., \& Groves, B. A. 2017, MNRAS, 466, 4403

Robitaille, T., \& Bressert, E. 2012, APLpy: Astronomical Plotting Library in Python, Astrophysics Source Code Library, , , 1208.017

Russell, S. C., \& Dopita, M. A. 1992, ApJ, 384, 508

Seab, C. G. 1987, in Astrophysics and Space Science Library, Vol. 134, Interstellar Processes, ed. D. J. Hollenbach \& H. A.

Thronson, Jr., 491-512

Seab, C. G., \& Shull, J. M. 1983, ApJ, 275, 652

Sipilä, O., Caselli, P., \& Juvela, M. 2017, A\&A, 601, A113

Sipilä, O., Harju, J., \& Juvela, M. 2011, A\&A, 535, A49

Smith, M. G., \& Weedman, D. W. 1971, ApJ, 169, 271

Spitzer, L. 1976, Comments on Astrophysics, 6, 177

Sutherland, R. S., \& Dopita, M. A. 2017, ApJS, 229, 34

Tuohy, I. R., Dopita, M. A., Mathewson, D. S., Long, K. S., \& Helfand, D. J. 1982, ApJ, 261, 473

Veilleux, S., \& Osterbrock, D. E. 1987, ApJS, 63, 295 
Vogt, F., \& Dopita, M. A. 2011, Ap\&SS, 331, 521

Vogt, F. P. A., Seitenzahl, I. R., Dopita, M. A., \& Ghavamian, P. 2017, A\&A, 602, L4

Welty, D. E., Lauroesch, J. T., Wong, T., \& York, D. G. 2016, ApJ, 821, 118
Westerlund, B. E., \& Mathewson, D. S. 1966, MNRAS, 131, 371 Williams, B. J., Borkowski, K. J., Reynolds, S. P., et al. 2006, ApJ, 652, L33

Xiao, X., \& Chen, Y. 2008, Advances in Space Research, 41, 416 
TABLE A1

Measured line fluXes for N132D Shocked Cloudlets P01 - P05

\begin{tabular}{|c|c|c|c|c|c|c|c|c|c|c|c|}
\hline Cloud ID\#: & & P01 & & P02 & & $\begin{array}{l}\text { P03 } \\
\end{array}$ & & P04 & & P05 & \\
\hline Lambda & ID & Flux & Error & Flux & Error & Flux & Error & Flux & Error & Flux & Error \\
\hline 3736.02 & {$[\mathrm{O} \mathrm{II}]$} & 176.1 & 10.4 & 102 & 7.9 & 247.2 & 23.0 & 119.1 & 2.7 & $\begin{array}{l}191.2 \\
\end{array}$ & 10.6 \\
\hline 3728.82 & {$\left[\begin{array}{lll}\mathrm{O} & \mathrm{II}\end{array}\right]$} & 92.8 & 9.8 & 79.4 & 8.7 & 278.4 & 23.4 & 46.1 & 1.7 & 59.6 & 6.5 \\
\hline 3835.4 & $\mathrm{H} \eta$ & 13.7 & 7.2 & 18.1 & 7.0 & 6.9 & 3.4 & 11.6 & 2.0 & & \\
\hline 3868.76 & [Ne III] & 19.1 & 2.3 & 27.0 & 2.3 & 60.8 & 4.2 & 21.7 & 1.4 & 33.5 & 2.8 \\
\hline 3888.8 & HeI, $\mathrm{H}$ & 27.0 & 5.9 & 29.4 & 4.0 & 15.5 & 3.9 & 19.6 & 1.4 & 22.8 & 2.5 \\
\hline 3933.66 & Ca II & 17.3 & 3.5 & 41.6 & 8.8 & 13.9 & 2.1 & 13.5 & 1.7 & 19.7 & 4.5 \\
\hline 3969.0 & {$[\mathrm{Ne} \mathrm{III}], \mathrm{Ca} \mathrm{II}, \mathrm{H} \epsilon$} & 42.1 & 4.2 & 44.4 & 5.1 & 41.5 & 5.5 & 29.3 & 1.6 & 31.2 & 3.4 \\
\hline 4068.6 & {$[\mathrm{~S}$ II] } & 34.6 & 1.9 & 27.9 & 1.5 & 33.8 & 2.7 & 31.6 & 0.8 & 35.3 & 2.3 \\
\hline 4076.35 & {$\left[\begin{array}{lll}\mathrm{S} & \mathrm{II}\end{array}\right]$} & 14.9 & 1.8 & 11.2 & 1.6 & 9.6 & 4.1 & 8.7 & 0.8 & 13.5 & 2.8 \\
\hline 4101.74 & $\mathrm{H} \delta$ & 28.8 & 2.2 & 27.4 & 2.1 & 22.3 & 2.4 & 21.4 & 1.3 & 32.9 & 3.9 \\
\hline 42 & {$[\mathrm{Fe} \mathrm{II}]$} & 7.6 & 2.5 & 3.2 & 0.9 & 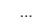 & & 4.8 & 0.5 & $\ldots$ & \\
\hline 42 & [Fe I & & & 2.3 & 1.3 & & & .5 & 0.8 & & \\
\hline 4287.43 & {$\left[\begin{array}{lll}\mathrm{Fe} & \mathrm{II}\end{array}\right]$} & 3.7 & 1.0 & 2.1 & 0.9 & 2.8 & 1.6 & 6.4 & 0.6 & & \\
\hline 4340.47 & $\mathrm{H} \gamma$ & 57.5 & 2.3 & 41.3 & 1.5 & 49.4 & 1.7 & 41.9 & 0.9 & 42.9 & 2.2 \\
\hline 4359.14 & {$[\mathrm{Fe} \mathrm{IX}]$} & 2.9 & 1.3 & & & & & & & & \\
\hline 4363.21 & {$\left[\begin{array}{lll}\mathrm{O} & \mathrm{III}\end{array}\right]$} & 9.9 & 2.8 & 8.5 & 1.5 & 14.6 & 3.4 & 8.0 & 1.8 & 7.6 & 1.2 \\
\hline $\begin{array}{l}4000.21 \\
4416.27\end{array}$ & [Fe II] & 5.0 & 1.7 & 8.2 & 1.7 & 7.7 & $\begin{array}{l}0.4 \\
1.9\end{array}$ & 7.3 & 0.9 & $\ldots$ & 1.2 \\
\hline 4452.38 & {$[\mathrm{Fe}$ II $]$} & 2.2 & 0.6 & - & & $\ldots$ & & 4.5 & 1.2 & $\ldots$ & \\
\hline 44 & {$[\mathrm{Fe}]$} & & & & & & & 1.3 & 0.3 & & \\
\hline 44 & $\mathrm{He}$ & 3.8 & 1.1 & 5.9 & 2.5 & 12.2 & 2.9 & 5 & 0.6 & 2.4 & 1.1 \\
\hline 4658.5 & [O III], [Fe II & 1.6 & 0.5 & 6.5 & 0.8 & 5.2 & 1.1 & 7.2 & 0.6 & 2.6 & 0.9 \\
\hline 4685.7 & He II & 7.0 & 1.9 & 9.4 & 1.1 & 3.2 & 1.2 & 6.8 & 0.6 & 5.6 & 1.6 \\
\hline 4701.53 & [Fe III] & 0.8 & 0.8 & & & $\ldots$ & & 3.4 & 0.6 & & \\
\hline 4754.68 & [Fe III] & 2.0 & 0.6 & 5.5 & 2.3 & . & & 1.4 & 0.6 & & \\
\hline 4814.54 & {$[\mathrm{Fe}$ II $]$} & 1.4 & 0.9 & 11.2 & 2.6 & $\cdots$ & & 4.3 & 0.5 & 2.4 & 1.6 \\
\hline 4861.29 & $\mathrm{H} \beta$ & 100.0 & 1.1 & 100.0 & 1.0 & 100.0 & 2.0 & 100.0 & 0.8 & 100.0 & 1.9 \\
\hline 4 & {$[\mathrm{Fe}]$} & 2.5 & 0.9 & 10.3 & 3.9 & & & 2.2 & 0.4 & $\ldots$ & \\
\hline 48 & {$[\mathrm{~F}$} & 1. & 0.6 & 6.8 & 2.1 & 3.5 & 1.4 & 8 & 0.5 & $\ldots$ & \\
\hline 4905.35 & {$[\mathrm{Fe} \mathrm{II}]$} & 1.2 & 0.9 & 4.8 & 1.1 & & & & & & \\
\hline 4958.91 & {$[\mathrm{O} \quad \mathrm{III}]$} & 20.7 & 1.3 & 27.0 & 1.3 & 74.3 & 2.3 & 31.7 & 0.6 & 49.1 & 1.6 \\
\hline 5006.84 & {$\left[\begin{array}{lll}\mathrm{O} & \mathrm{III}\end{array}\right]$} & 52.6 & 1.5 & 97.9 & 1.4 & 198.4 & 4.1 & 95.1 & 1.0 & 157.1 & 1.7 \\
\hline 5111.64 & {$[\mathrm{Fe} \mathrm{II}]$} & 0.1 & 0.5 & & & & & 4.1 & 1.2 & & \\
\hline 5158.79 & {$[\mathrm{Fe} \mathrm{II}],[\mathrm{Fe} \mathrm{VII}]$} & 4.8 & 0.9 & 11.2 & 0.9 & 10.3 & 1.4 & 18.5 & 0.9 & 11.5 & 1.8 \\
\hline 51 & {$[\mathrm{~N} \mathrm{I}]$} & 0.6 & 0.5 & 5.6 & 1.7 & & & 11.5 & 4.7 & & \\
\hline 52 & {$[\mathrm{~F}$} & 1. & 0.8 & 5.0 & 1.5 & 1.4 & 0.7 & 6.9 & 0.6 & 4.2 & 1.3 \\
\hline 5270.4 & [Fe III] & 2.0 & 1.1 & & & $\ldots$ & & 8.6 & 2.4 & 6.4 & 2.2 \\
\hline 5273.36 & {$[\mathrm{Fe} \mathrm{II}]$} & 2.1 & 2.0 & 39.3 & 17.8 & & & & & & \\
\hline 5303.06 & [Fe XIV] & 2.4 & 1.2 & 12.0 & 1.3 & 2.4 & 0.8 & 5.5 & 0.6 & 1.5 & 0.6 \\
\hline 5334.5 & {$[\mathrm{Fe} \mathrm{II}],[\mathrm{Fe} \mathrm{VI}]$} & 1.2 & 0.6 & 6.6 & 2.4 & $\ldots$ & & 4.5 & 0.5 & 4.2 & 1.4 \\
\hline 5376.0 & {$[\mathrm{Fe} I I],[\mathrm{Fe}$ VIII] } & 3.5 & 1.5 & & & $\ldots$ & & 2.7 & 0.4 & & \\
\hline 5527.3 & {$[\mathrm{Fe}$ II $]$} & $\ldots$ & & $\ldots$ & & & & 2.4 & 0.7 & 3.1 & 1.4 \\
\hline 5754.59 & {$[\mathrm{~N} \mathrm{II}]$} & & & & & 0.7 & 0.3 & 1 & 0.2 & & \\
\hline & & 7.4 & 1.1 & 9.3 & 1.2 & 8.0 & 0.9 & & & 7.4 & 0.9 \\
\hline 6086.97 & {$[\mathrm{Fe} \mathrm{VII}]$} & & & & & & & 2.2 & 0.4 & 2.2 & 1.0 \\
\hline 6300.3 & {$[\mathrm{O} \mathrm{I}]$} & 87.7 & 3.5 & 106 & 4.3 & 62.3 & 1.9 & 90.8 & 1.5 & 73.6 & 3.6 \\
\hline 6312.06 & [S III] & & & 2.0 & 1.0 & & & 0.8 & 0.4 & 0.5 & 0.2 \\
\hline 6363.78 & 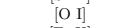 & 29.4 & 1.4 & 17.8 & 1.3 & 26 & 1.5 & 30.7 & 0.4 & 22.8 & 2.4 \\
\hline 63 & {$[\mathrm{~F}$} & 2.3 & 0.5 & 6.5 & 1.3 & 3.9 & 1.2 & 4.2 & 0.4 & & \\
\hline 65 & {$\left[\begin{array}{lll}\mathrm{N} & \mathrm{II}\end{array}\right]$} & 28.2 & 1.3 & 19.6 & 0.8 & 32.6 & 2.3 & 29.9 & 0.5 & 28.3 & 1.1 \\
\hline & & 302.3 & 1.4 & 301.2 & 1.9 & 300.9 & 3.1 & 299.5 & 0. & 299.5 & 1.6 \\
\hline & & 85.0 & 0 & 67.0 & 0.8 & 98.9 & 1.5 & 94.8 & & 87.0 & 1.2 \\
\hline 66 & 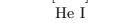 & 2. & 0.4 & 3.8 & .2 & 2.6 & 0.4 & .0 & .2 & 1.7 & 0.9 \\
\hline 67 & 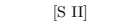 & 70.6 & 0 & 43 & 18 & 83.1 & 2.0 & 60.3 & .4 & 67.6 & 0.9 \\
\hline 6730 & & 124.1 & 1.0 & 78.9 & 19 & 128.7 & 2.4 & 113.0 & 0.3 & 112.0 & 1.0 \\
\hline & & 4. & 0.5 & 4.4 & 1.1 & 3.9 & 0.5 & 5.3 & 0.2 & 5.1 & 0.7 \\
\hline $713:$ & & 1.4 & 0.2 & 4.9 & 0.7 & 6.0 & 0.5 & 4.5 & 0.2 & 4.9 & 0.4 \\
\hline 7155.1 & {$[\mathrm{~F}$} & 15.3 & 0.4 & 16.1 & 0.6 & 13.4 & 0.6 & 26.7 & 0.3 & 13.1 & 0.4 \\
\hline 71 & & 1. & & r & & $=$ & & & & 20 & 0.3 \\
\hline & & & & & & & & & & & 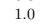 \\
\hline 73 & {$[\mathrm{Ca} \mathrm{II}],[\mathrm{O}$} & 4 & 1 & & 2 & 38 & 2 & 51 & 6 & & 1.8 \\
\hline 73 & [OU] & 11 & 0. & 17 & 1.3 & 18.6 & 1.7 & 18 & & 23.6 & 1.8 \\
\hline & & 8 & 0 & 8 & 1.1 & 7.1 & 0.9 & 11 & 0.3 & 4.1 & 0.6 \\
\hline 7388 & & 2. & 0 & 4.6 & 1.3 & $\ldots$ & & 3.4 & 0.3 & $\ldots$ & \\
\hline 7411.61 & & 1.1 & 0.4 & 1.7 & 0.8 & & & 0 & 0.4 & & \\
\hline 7452 & {$[\mathrm{Fe}]$} & 5.3 & 0.3 & 3.9 & 0.6 & 4.6 & 0.6 & 1 & 0 & 2.6 & 0.5 \\
\hline 7637 & {$[\mathrm{Fe}]$} & 1.9 & 0.5 & 2.5 & 1.0 & 4.3 & 0.7 & & 0.3 & 1.4 & 1.2 \\
\hline 7665 & F & & & & & & & & 3 & & \\
\hline & & 1.0 & 0.3 & 3.7 & 1.1 & 1.7 & 0.7 & & & & \\
\hline & & 2.4 & 0.8 & 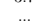 & & 3.8 & 0.9 & & & $\ldots$ & \\
\hline 812 & & $\ldots$ & & $\ldots$ & & & & 2.4 & 0.4 & & \\
\hline 8229. & & & & & & & & 1.1 & 0.2 & 0.5 & 0.3 \\
\hline 8234.54 & [Fe I & 0.8 & 0.3 & 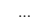 & & 2.5 & 0.4 & 3.7 & 0.3 & & \\
\hline 8542.1 & $\mathrm{Ca}$ & 3.1 & 0.7 & & & 3.3 & 0.7 & 3.7 & 0.6 & 4.2 & 1.0 \\
\hline & {$[\mathrm{Cl}$} & 0.5 & 0.3 & & & & & 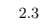 & 0.5 & & \\
\hline & & 19 & 0.9 & 20.6 & 1.4 & 20.7 & 1.1 & 3 & 0.6 & 25.7 & 2.8 \\
\hline & & 0. & & 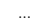 & & 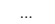 & & & & & \\
\hline & & & & & & & & 1.3 & 0.2 & & \\
\hline & [Fe II] & 7.7 & 1.0 & 5.3 & 4.2 & 5.2 & 3.5 & 9.5 & 0.6 & - & \\
\hline $\mathrm{F}(\mathrm{H} \beta) \times 1$ & $\left(\mathrm{erg} / \mathrm{cm}^{2} / \mathrm{s}\right)$ & 86.17 & & 45.67 & & 49.32 & & 106.87 & & 38.71 & \\
\hline
\end{tabular}

\section{APPENDIX}

In the following four Tables, A1 through A4, we present the measured line fluxes of the 20 shocked ISM clouds in N132D. No reddening correction has been applied, since the mean Balmer Decrement as measured; $\mathrm{H} \alpha / \mathrm{H} \beta / \mathrm{H} \gamma / \mathrm{H} \delta$ $=298.7 / 100.0 / 44.7 / 23.9$ is indistinguishable (within the errors) with the theoretical Balmer Decrement for $\log n_{e}=$ $10^{4} \mathrm{~cm}^{-3}$, and $T_{e}=5000 \mathrm{~K} ; 300 / 100 / 46.0 / 25.3$. On the basis of the soft X-ray absorption Borkowski et al. (2007) determined a best-fit hydrogen column density of $1.4 \times 10^{20} \mathrm{~cm}^{-2}$ in the western part of N132D and $1.4-4.1 \times 10^{21}$ $\mathrm{cm}^{-2}$ in the south. Assuming dust is not destroyed in the bar of the LMC, and using the Fitzpatrick (1986) $N_{\mathrm{H}} / E_{\mathrm{B}-\mathrm{V}}$ conversion factor, these figures would imply a reddening of $E_{\mathrm{B}-\mathrm{V}}=0.005$ mag. and 0.06-0.17 mag., respectively. 
TABLE A2

Measured line fluXes for N132D Shocked Cloudlets P06 - P10

\begin{tabular}{|c|c|c|c|c|c|c|c|c|c|c|c|}
\hline Cloud ID\#: & & P06 & & P07 & & P08 & & P09 & & P10 & \\
\hline Lambda & ID & Flux & Error & Flux & Error & Flux & Error & Flux & Error & Flux & Error \\
\hline 3736.02 & {$[\mathrm{O} \mathrm{II}]$} & 174.3 & 7.4 & 93.6 & 29.2 & 67.3 & 7.6 & 77.2 & 15.5 & 138.2 & 8.3 \\
\hline 3728.82 & {$\left[\begin{array}{lll}\mathrm{O} & \mathrm{II}\end{array}\right]$} & 61.8 & 5.9 & 54.9 & 28.8 & 33.1 & 5.3 & & & 120.4 & 8.3 \\
\hline 3835.4 & $\mathrm{H} \eta$ & 12.2 & 2.7 & 6.6 & 1.8 & 14.5 & 4.8 & 16.0 & 3.5 & & \\
\hline 3868.76 & [Ne III] & 16.5 & 3.1 & 19.7 & 2.0 & 12.7 & 9.3 & & & 34.8 & 3.7 \\
\hline 3888.8 & HeI, $\mathrm{H}$ & 24.0 & 3.6 & 14.2 & 2.3 & 14.4 & 3.5 & & & 6.6 & 2.8 \\
\hline 3933.66 & Ca II & 15.0 & 2.1 & 14.6 & 3.6 & 14.3 & 5.6 & 23.2 & 7.8 & & \\
\hline 3969 & {$[\mathrm{Ne}$ III], Ca II, He } & 30.5 & 3.3 & 21.8 & 2.0 & 37.3 & 5.4 & $\ldots$ & & 25.8 & 4.2 \\
\hline 4026.21 & He I & & & 4.2 & 1.5 & & & & & 5.2 & 1.1 \\
\hline 4068.6 & {$[\mathrm{~S} \mathrm{II}]$} & 33.4 & 1.7 & 28.2 & 1.1 & 15.6 & 3.3 & 21.9 & 2.6 & 25.0 & 1.6 \\
\hline 4076.35 & {$\left[\begin{array}{ll}\mathrm{S} \\
\mathrm{II}\end{array}\right]$} & 16.0 & 1.9 & 7.2 & 1.0 & 5.5 & 2.3 & 14.7 & 2.4 & 11.0 & 2.0 \\
\hline 4101.74 & $\mathrm{H} \delta$ & 25.3 & 2.6 & 17.9 & 1.2 & 38.5 & 5.1 & 22.0 & 3.5 & 29.4 & 2.3 \\
\hline 4244 & [Fe II] & $\ldots$ & & 3.9 & 0.9 & $\ldots$ & & $\ldots$ & & 6.1 & 2.7 \\
\hline 4276.83 & {$[\mathrm{Fe}$ II] } & $\ldots$ & & 2.9 & 0.8 & $\ldots$ & & $\ldots$ & & $\ldots$ & \\
\hline 4287.43 & {$\left[\begin{array}{l}\mathrm{Fe} \\
\mathrm{FI}]\end{array}\right.$} & $\cdots$ & & 1.5 & 0.6 & $\cdots$ & & $\cdots$ & & $\cdots$ & \\
\hline 4340.47 & $\mathrm{H} \gamma$ & 46.0 & 1.7 & 51.9 & 2.6 & 53.1 & 3.1 & 38.7 & 2.0 & 41.5 & 1.3 \\
\hline 4359.14 & {$[\mathrm{Fe} \mathrm{IX]}$} & 8.3 & 5.6 & & & 5.3 & 2.6 & $\ldots$ & & 2.2 & 0.9 \\
\hline $\begin{array}{l}4303.14 \\
4363.21\end{array}$ & [O III] & 5.1 & 6.2 & 8.4 & 1.9 & 7.1 & 1.5 & $\ldots$ & & 11.0 & 2.0 \\
\hline 4416.27 & {$[\mathrm{Fe} \mathrm{II}]$} & 3.5 & 2.0 & 6.3 & 1.7 & & & & & & \\
\hline 4452.38 & {$[\mathrm{Fe}$ II] } & & & 4.5 & 0.7 & $\cdots$ & & $\cdots$ & & 2.0 & 1.0 \\
\hline 4471.5 & $\mathrm{HeI}$ & 1.1 & 0.4 & 1.9 & 0.5 & & & & & 3.6 & 1.1 \\
\hline 4658.5 & [O III], [Fe III] & 7.5 & 0.8 & 3.3 & 0.6 & 3.8 & 0.9 & 4.1 & 1.5 & 4.9 & 1.1 \\
\hline 4685.7 & $\mathrm{He}$ II & 8.2 & 1.1 & 6.2 & 0.7 & 1.4 & 0.6 & $\ldots$ & & 8.6 & 1.4 \\
\hline 4701.53 & [Fe III $]$ & $\ldots$ & & 2.4 & 0.7 & 5.9 & 1.1 & $\ldots$ & & $\ldots$ & \\
\hline 4754.68 & [Fe III] & & & 4.6 & 1.9 & & & & & & \\
\hline 4814.54 & {$[\mathrm{Fe} \mathrm{II}]$} & 4.4 & 0.9 & 2.5 & 0.5 & & & & & & \\
\hline 4861.29 & $\mathrm{H} \beta$ & 100.0 & 1.0 & 100.0 & 6.4 & 100.0 & 2.4 & 100.0 & 1.5 & 100.0 & 1.7 \\
\hline 4881 & [Fe III] & 4.0 & 0.7 & $\ldots$ & & & & & & 0.7 & 0.5 \\
\hline 488 & {$[\mathrm{Fe}]$} & & & & & 2.5 & 1.1 & 2.3 & 0.8 & & \\
\hline 49 & {$[\mathrm{~B}$} & 4.6 & 1.2 & & & 4.2 & 2.9 & & & & \\
\hline 4958.91 & {$\left[\begin{array}{lll}1 & \text { III] }\end{array}\right]$} & 15.0 & 1.3 & 31.9 & 5.1 & 14.5 & 14.7 & & & 47.6 & 1.6 \\
\hline 5006.84 & [O III] & 61.2 & 1.1 & 115.3 & 6.3 & 43.3 & 1.4 & & & 136.6 & 1.6 \\
\hline 5158.79 & {$[\mathrm{Fe} \mathrm{II}],[\mathrm{Fe} \mathrm{VII}]$} & 15.2 & 1.4 & 11.3 & 0.5 & 15.6 & 1.7 & 14.4 & 1.0 & 5.0 & 1.1 \\
\hline 5198.8 & {$[\mathrm{~N} \mathrm{I}]$} & 2.2 & 1.2 & 0.4 & 0.2 & & & & & $\ldots$ & \\
\hline 5261.66 & {$[\mathrm{Fe}$ II] $]$} & 5.5 & 0.9 & 5.3 & 0.5 & 2.8 & 0.8 & 6.8 & 1.1 & .... & \\
\hline 5270.4 & [Fe III] & 3.3 & 2.0 & 1.0 & 0.5 & 列 & & & & $\ldots$ & \\
\hline $527:$ & {$[\mathrm{Fe}$} & & & 0.5 & 0 & & & & & & \\
\hline 5303.06 & [Fe XI & 6.6 & 1.5 & 5.5 & 0.9 & 6.7 & 2.6 & 9.1 & 1.1 & 6.0 & 1.3 \\
\hline 5334.5 & {$[\mathrm{Fe} \mathrm{II}],[\mathrm{Fe} \mathrm{VI}]$} & $\ldots$ & & 0.0 & & $\begin{array}{l}.01 \\
4.9\end{array}$ & $\begin{array}{l}2.0 \\
2.0\end{array}$ & $\begin{array}{l}9.1 \\
3.1\end{array}$ & $\begin{array}{l}1.1 \\
0.7\end{array}$ & 0.0 & 1.0 \\
\hline 5376 & [Fe III],[Fe VIII] & & & 1.4 & 1.1 & $\ldots$ & & & & 3.1 & 1.6 \\
\hline 5527.3 & {$[\mathrm{Fe}$ II] } & 1.6 & 2.1 & & & & & & & & \\
\hline 5754.59 & {$[\mathrm{~N}$ II] } & 2.9 & 1.2 & & & & & & & & \\
\hline 5875.61 & $\mathrm{He} \mathrm{I}$ & 9.4 & 1.1 & 5.2 & 2.5 & 6.1 & 3.3 & 12.3 & 1.0 & 5.9 & 1.4 \\
\hline & {$[\mathrm{Fe} \mathrm{VII}]$} & 1.4 & 0.4 & 1.4 & 0.5 & & & 1.7 & 8 & 2.7 & 0.6 \\
\hline & & 86.7 & 5.8 & 58.8 & 0.9 & 57.3 & 9.4 & 96.7 & & 71.3 & 1.0 \\
\hline & & . & 0.5 & & & & & 8.1 & 4 & & \\
\hline 6363.78 & {$\left[\begin{array}{lll}\mathrm{O} & \mathrm{I}\end{array}\right]$} & 29.1 & 2.1 & 20.8 & 0.9 & 20.4 & 8.9 & 30.6 & 0.8 & 24.8 & 0.8 \\
\hline 6374.52 & {$[\mathrm{Fe} \mathrm{X}]$} & 3.2 & 1.2 & & & & & & & 4.2 & 0.7 \\
\hline 654 & {$\left[\begin{array}{lll}\mathrm{N} & \mathrm{II}\end{array}\right]$} & 26.0 & 0.9 & 25.2 & 1.3 & 19.4 & 2.2 & 26.1 & 1.9 & 24.7 & 1.4 \\
\hline 656 & $\mathrm{H}$ & 319.0 & 1.4 & 298.7 & 2.7 & 298.2 & 4.1 & 300.0 & 2.5 & 300.6 & 1.7 \\
\hline 658 & {$[\mathrm{~N}$} & 78.3 & 0.8 & 71.8 & 0.7 & 53.9 & 2.1 & 82.8 & 1.2 & 83.7 & 0.8 \\
\hline 6678. & $\mathrm{He}$ & 2.0 & 0.5 & & & 3.6 & 0.6 & 3.7 & .5 & 3.1 & .7 \\
\hline 67 & [S & 42. & 0. & 59.8 & 0 & 57.1 & & 80.2 & & 43.8 & .6 \\
\hline & [s & 73.8 & 0.6 & 106.7 & 0 & 97.0 & 0.9 & 124.5 & & 73.5 & 17 \\
\hline 706 & $\mathrm{He}$ I & 4.6 & 0.5 & 5.2 & 0.5 & 7.6 & 1.2 & 9.6 & .5 & 1 & 6 \\
\hline 713 & {$\left[\begin{array}{ll}\mathrm{Ar} & \mathrm{III}\end{array}\right]$} & 1. & 0.4 & 3. & 0.5 & 2.1 & 0.6 & 3.9 & .5 & 9 & 0.5 \\
\hline 715 & & 22.4 & 0.6 & 15.9 & 0. & 19.4 & 0.9 & 21.4 & 6 & 12.0 & .6 \\
\hline 7172 & & 3.8 & 0.5 & 2.4 & 0.4 & 2.0 & 0.5 & 2.6 & .5 & 3.8 & .7 \\
\hline 7291.47 & {$[\mathrm{Ca}$} & 39.9 & 0.9 & 35.0 & 2.7 & 44.2 & 2.1 & 45.8 & .1 & 12.6 & 1.2 \\
\hline 7321 & $\mathrm{Ca} I \mathrm{II}],[\mathrm{O} \mathrm{II}]$ & 57.6 & 3.0 & 39.0 & 1. & & & 47.3 & 6 & 46.6 & 4.0 \\
\hline 733 & [O & 13.2 & 1. & 14.5 & 0 & & & 4.2 & & 35.9 & 4 \\
\hline & & 12 & 0 & 5. & 0 & 11.8 & 1.4 & 10.8 & 3 & 5.6 & 1.0 \\
\hline 738 & {$[\mathrm{Fe}$} & 6 & 1.3 & 4.2 & 7. & 4.1 & 1.3 & & & & \\
\hline & & 1. & 0.4 & & & & & 1.7 & 0.5 & & \\
\hline & & 9.6 & 0.9 & 5.6 & 0.7 & 7.1 & 1.1 & 4.5 & 0.6 & 3.1 & 0.5 \\
\hline 7637. & & 4.0 & 0.6 & 2.0 & 0.8 & 7.2 & 1.5 & 5.4 & 1.1 & & \\
\hline 7665. & {$[\mathrm{Fe}$} & & & & & 1.2 & & & & & \\
\hline 7686.93 & [Fe II] & 1.7 & 0.6 & 1.0 & 0.4 & $\ldots$ & & 1.3 & 0.4 & 1.3 & 0.5 \\
\hline 78 & & 1.9 & 0.7 & & & & & & & $\ldots$ & \\
\hline & & & & 1.5 & 0.4 & 3.6 & 0.9 & & & $\ldots$ & \\
\hline & & 1.2 & 0.2 & & & 0.8 & 0.4 & 3.0 & 8 & & \\
\hline & & 3.6 & 0.4 & 2 & 0.8 & 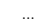 & & 2.7 & 6 & $\ldots$ & \\
\hline & & & & 4.9 & 1. & & & 1.4 & 0.4 & & \\
\hline 8578.69 & {$\left[\begin{array}{lll}\mathrm{Cl} & \mathrm{II}\end{array}\right]$} & $\ldots$ & & 1.9 & 0.3 & $\ldots$ & & & & $\ldots$ & \\
\hline 8616.95 & {$[\mathrm{Fe} \mathrm{II}]$} & 29.0 & 1.4 & 19.0 & 0.6 & 19.1 & 2.7 & 26.1 & & 11.0 & 1.4 \\
\hline 8662.1 & {$\left[\begin{array}{lll}\mathrm{Ca} & \mathrm{II}\end{array}\right]$} & & & 1.8 & 0.6 & & & 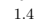 & 0.5 & & \\
\hline 87 & {$[\mathrm{C}$} & 1. & 0.6 & 1.3 & 0 & & & & & 5.7 & 1.6 \\
\hline & $\mathrm{H}$ & & & & & & & 4 & .9 & & \\
\hline & & 5 & 1.5 & 4.5 & 3.5 & & & 6.9 & 3.0 & 4.2 & 1.1 \\
\hline$\frac{F}{\mathrm{~F}(\mathrm{H} \beta) \times 1 \mathrm{l}}$ & $\frac{[\mathrm{rem}]}{\left(\mathrm{erg} / \mathrm{cm}^{2} / \mathrm{s}\right)}$ & $\begin{array}{r}0.0 \\
48.9 \\
\end{array}$ & & $\frac{4.0}{76.5}$ & & $\frac{1}{76.5}$ & & 56.8 & & $\begin{array}{l}4.2 \\
54.8\end{array}$ & \\
\hline
\end{tabular}


TABLE A3

Measured line fluxes for N132D Shocked Cloudlets P11 - P15

\begin{tabular}{|c|c|c|c|c|c|c|c|c|c|c|c|}
\hline Cloud ID\#: & & P11 & & P12 & & P13 & & P14 & & P15 & \\
\hline Lambda & ID & Flux & Error & Flux & Error & Flux & Error & Flux & Error & Flux & Error \\
\hline 3736.0 & [O II] & 88.5 & 17.4 & 216.6 & 4.5 & 223.3 & 12.4 & 229.5 & 2.0 & 295.7 & 6.7 \\
\hline 3728.8 & [O II] & 197.4 & 24.0 & 106.7 & 4.4 & 148.8 & 12.4 & 119.5 & 2.0 & 208.4 & 6.7 \\
\hline 3835.4 & $\mathrm{H} \eta$ & & & 6.5 & 0.7 & & & 5.5 & 0.5 & 8.2 & 0.9 \\
\hline 3868.8 & {$[\mathrm{Ne} I I I]$} & 41.7 & 4.1 & 29.9 & 0.5 & 23.3 & 2.8 & 32.2 & 0.5 & 35.6 & 0.8 \\
\hline 3888.8 & $\mathrm{HeI}, \mathrm{H}$ & 14.2 & 5.7 & 15.9 & 0.7 & 11.5 & 2.8 & 19.8 & 0.4 & 21.8 & 0.8 \\
\hline 3933.7 & Ca II & & & 11.8 & 0.4 & $\ldots$ & & 16.8 & 0.4 & 13.9 & 1.1 \\
\hline 3969.0 & {$[\mathrm{Ne} \mathrm{III}], \mathrm{Ca} \mathrm{II}, \mathrm{H} \epsilon$} & 41.6 & 10.9 & 30.8 & 0.7 & $\ldots$ & & 32.5 & 0.6 & 34.8 & 1.2 \\
\hline 4026.2 & He I & 11.7 & 3.5 & 2.0 & 0.5 & & & 1.6 & 0.3 & 1.2 & 0.4 \\
\hline 4068.6 & [S II] & 31.7 & 3.7 & 27.5 & 0.4 & 22.8 & 2.2 & 41.4 & 0.3 & 29.2 & 0.7 \\
\hline 4076.4 & [S II] & 15.4 & 2.4 & 9.2 & 0.3 & 3.0 & 1.2 & 14.1 & 0.3 & 9.7 & 0.6 \\
\hline 4101.7 & $\mathrm{H} \delta$ & 23.0 & 3.6 & 22.2 & 0.5 & 12.2 & 2.5 & 24.5 & 0.3 & 28.0 & 0.6 \\
\hline 4244 & {$[\mathrm{Fe} \mathrm{II}]$} & & & 4.3 & 0.2 & & & 4.9 & 2 & .8 & 0.5 \\
\hline 4276.8 & {$[\mathrm{Fe}$ II] } & 8.5 & 2.4 & 0.9 & 0.2 & & & 1.5 & 0.2 & 2.4 & 0.7 \\
\hline 4287.4 & {$[\mathrm{Fe} \mathrm{II}]$} & $\ldots$ & & 3.0 & 0.2 & 2.7 & 1.5 & 3.9 & 2 & 3.6 & 0.5 \\
\hline 4319.6 & {$[\mathrm{Fe} \mathrm{II}]$} & $\ldots$ & & 0.5 & 0.1 & & & 0.7 & 0.2 & $\ldots$ & \\
\hline 4340.5 & $\mathrm{H} \gamma$ & 53.6 & 2.4 & 42.0 & 0.6 & 38.9 & 1.5 & 44.8 & 0.4 & 48.7 & 0.6 \\
\hline 4359.1 & {$[\mathrm{Fe}$ IX] } & & & 2.7 & 0.5 & & & 4.1 & 0.4 & 3.7 & 0.6 \\
\hline 4363.2 & {$\left[\begin{array}{ll}\mathrm{O} & \mathrm{III}\end{array}\right]$} & 14.3 & 1.9 & 7.8 & 0.4 & 7.8 & 1.4 & 7.1 & 0.3 & 7.2 & 0.5 \\
\hline 4416.3 & {$[\mathrm{Fe}]$} & $\ldots$ & & 4.7 & 0.4 & & & 4.9 & 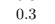 & 7.2 & 0.6 \\
\hline 44 & F & $\ldots$ & & 0.6 & 0.1 & ... & & 1.0 & 0.1 & 4 & 3 \\
\hline 4458.0 & {$[\mathrm{Fe}$ II] } & $\ldots$ & & 1.1 & 0.2 & & & 0.9 & 2 & 1.2 & 0.5 \\
\hline 4471.5 & $\mathrm{He} \mathrm{I}$ & 3.6 & 1.2 & 3.8 & 0.3 & 5.2 & 1.6 & 3.9 & 2 & 4.5 & 0.4 \\
\hline 4566.8 & $\mathrm{Mg} \mathrm{I}]$ & $\ldots$ & & 2.5 & 0.5 & & & 3.6 & 0.2 & 1.8 & 0.4 \\
\hline 4658.5 & $\mathrm{IIII},[\mathrm{Fe}$ & 2.9 & 1.0 & 5.0 & 0.2 & 3.4 & 0.8 & 5.1 & 0.1 & 7.0 & 0.3 \\
\hline 4685.7 & $\mathrm{He}$ II & $\ldots$ & & 6.5 & 0.2 & 3.8 & 1.2 & 5.5 & 0.2 & 5.9 & 0.3 \\
\hline 4701.5 & [Fe III] & $\ldots$ & & 4.1 & 1.3 & & & 1.1 & 0.1 & 1.9 & 0.3 \\
\hline 47 & {$[\mathrm{~F}$} & ... & & 0. & 0. & & & 0 & & & 3 \\
\hline 48 & {$[\mathrm{Fe}$} & $\ldots$ & & 2.3 & 0.2 & 5.1 & 2.3 & 2.4 & & 1 & .2 \\
\hline 4861.3 & $\mathrm{H} \beta$ & 100.0 & 3.1 & 100.0 & 1.2 & 100.0 & 1.1 & 100.0 & 3 & 100.0 & 7 \\
\hline 4881.0 & {$[\mathrm{Fe} I \mathrm{III}]$} & $\ldots$ & 0.1 & 2.2 & 0.9 & $\ldots$ & 1.1 & 2.1 & 0.2 & 2.9 & 0.4 \\
\hline 4889.6 & {$[\mathrm{Fe} \mathrm{II}]$} & $\ldots$ & & 2.0 & 0.9 & $\ldots$ & & 2.2 & 0.2 & 1.7 & 0.4 \\
\hline 4905.4 & {$[\mathrm{Fe}$ II] } & $\ldots$ & & 0.9 & 0.9 & & & 0.9 & 0.2 & 0.6 & 0.2 \\
\hline 4958.9 & {$\left[\begin{array}{lll}\mathrm{O} & \mathrm{III}\end{array}\right]$} & 76.7 & 19.7 & 39.3 & 1.2 & 35.5 & 1.1 & 38.4 & 0.3 & 33.9 & 0.6 \\
\hline 5006.8 & {$\left[\begin{array}{lll}\mathrm{O} & \mathrm{III}\end{array}\right]$} & 249.2 & 4.5 & 120.5 & 1.5 & 116.1 & 1.4 & 114.9 & 8 & 103.6 & 1.3 \\
\hline 5111.6 & {$[\mathrm{Fe}$ II] } & - & & 2.8 & 1.0 & & & 1.6 & 1 & 1.0 & 0.2 \\
\hline 5158 & $\mathrm{II}],[\mathrm{F}$ & 7.2 & 1.8 & 11.3 & 0.5 & 14.9 & 1.1 & 11.7 & 0.2 & .7 & .2 \\
\hline 5198.8 & 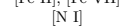 & 1.4 & 0.6 & 4.2 & 0.7 & 18.1 & 2.5 & 3.1 & 0.3 & 2.9 & 0.4 \\
\hline 5261.7 & {$[\mathrm{Fe} \mathrm{II}]$} & 4.5 & 1.3 & 4.1 & 0.4 & 2.4 & 0.7 & 4.2 & 0.1 & 3.8 & 0.3 \\
\hline 5270.4 & [Fe III] & 6.1 & 1.2 & 3.7 & 0.9 & & & 0.9 & 1.3 & & \\
\hline 5273.4 & {$[\mathrm{Fe}$ II] } & 3.4 & 1.4 & 1.2 & 0.8 & & & 3.3 & 1.4 & 4.6 & 0.4 \\
\hline 5303.1 & {$[\mathrm{Fe} \mathrm{XIV}]$} & 30.4 & 1.9 & 3.2 & 0.6 & 4.3 & 1.5 & 3.7 & 0.2 & 1.6 & 0.3 \\
\hline 5334.5 & {$[\mathrm{Fe} \mathrm{II}],[\mathrm{Fe} \mathrm{VI}]$} & 5.1 & 1.8 & 2.0 & 0.5 & 1.5 & 0.7 & 2.2 & 0.1 & 1.7 & 0.2 \\
\hline 5376.0 & {$[\mathrm{Fe} \mathrm{II}],[\mathrm{Fe} \mathrm{V}$} & & & 0.5 & 0. & & & & & & 1.1 \\
\hline 5527 & & $\ldots$ & & 2.5 & & & & & & & 3 \\
\hline 5754. & & $\ldots$ & & 1.2 & 0.3 & 4.1 & 0.9 & 1.3 & 1 & 1.8 & .2 \\
\hline 5875.6 & He I & 10.2 & 1.9 & 8.0 & 0.4 & 9.1 & 0.8 & 11.5 & 0.1 & 10.4 & 0.3 \\
\hline 6087.0 & {$[\mathrm{Fe} \mathrm{VII}]$} & 1.3 & 0.7 & 1.2 & 0.5 & & & 0.6 & 0.1 & 1.6 & 0.2 \\
\hline 630 & {$[0]$} & 43.5 & 1.0 & 49.5 & 1.5 & 65.5 & 3.3 & 104.5 & 1.3 & 79.0 & 0.5 \\
\hline 631 & {$[\mathrm{~S}]$} & & & & & 1.4 & 1.0 & 0.9 & 0.4 & 1.2 & 0.7 \\
\hline 636 & {$[\mathrm{O}$} & 16.3 & 0.8 & 12.4 & 1.2 & 22.6 & 1.3 & 35.2 & 3 & 26.8 & 0.4 \\
\hline 637 & {$[\mathrm{Fe}$} & 13.3 & 1.0 & & & 6.0 & 2.2 & & & 3.7 & 0.3 \\
\hline & {$[\mathrm{N}$} & & 1.9 & 24 & 1.0 & 63.3 & 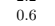 & 29 & & 30.2 & 5 \\
\hline 656 & $\mathrm{H}_{c}$ & 296.9 & 3.0 & 298.0 & 2. & 295.6 & 7 & 05.4 & 3 & 297.6 & 9 \\
\hline 6583.5 & {$[\mathrm{~N}]$} & 52.9 & 1.2 & 70.8 & 0. & 197.1 & 1.1 & 91.4 & 4 & 93.6 & .6 \\
\hline 6678.2 & $\mathrm{He}$ & 2.7 & 0.4 & 1.8 & 0.3 & 1.9 & 0.4 & 3.2 & 1 & 2.7 & 1.1 \\
\hline 671 & & 31.2 & 0.8 & 61.8 & 0.5 & 82.3 & 0.7 & 85.8 & .3 & 94.3 & 13 \\
\hline 673 & [S & 61.4 & 0.8 & 107.5 & 0.5 & 119.5 & 0.9 & 144.9 & 0.4 & 134.9 & 0.4 \\
\hline 7065.3 & $\mathrm{He}$ & 4.1 & 0.7 & 3.3 & 0. & 4.0 & 4 & 5.2 & 0.1 & 4.0 & 0.2 \\
\hline 7135 & {$[\mathrm{Ar} \mathrm{I}$} & 6.8 & 0 & 3. & 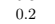 & 100 & & & & & 2 \\
\hline 71 & & & & 13 & & 13 & & 22 & & & 2 \\
\hline 717 & & 2. & & 3 & & 2. & & & & & 2 \\
\hline 729 & [Ca II] & 21.0 & 0. & $\begin{array}{l}0.4 \\
30.8\end{array}$ & 3. & 26.3 & 5 & 39.1 & 3 & 26.9 & 4 \\
\hline 732 & $\mathrm{II}]$, & 35.5 & 1. & 38.2 & 1. & 32.3 & 1.5 & 51. & 8 & 36.0 & 17 \\
\hline 733 & & 23.9 & 1.2 & 14.5 & 1.1 & 11.5 & 0.9 & 18.8 & 5 & 14.1 & .5 \\
\hline 7377 & & 6.8 & 0.9 & 5.3 & 0.5 & 6.6 & 0.7 & 10.2 & 1 & 8.8 & 0.3 \\
\hline 7388 & ST & 1.8 & 0.5 & 1.7 & 0.4 & & & 2 & 1 & .2 & 0.2 \\
\hline 7411. & [N & 53.8 & 25.0 & & & 0.8 & 2 & & 1 & 8 & 2.2 \\
\hline 7452 & & 5.9 & 0.8 & 4.9 & 0. & . & & & & & 2 \\
\hline & & $\ldots$ & & 1.4 & & 0. & & & & & \\
\hline & & -... & & 1.4 & & 0.5 & 3 & 8 & & .5 & 2 \\
\hline & & $\ldots$ & & 1.2 & 0.3 & 1.3 & 0.5 & 0 & 1 & 1.5 & .2 \\
\hline 789 & & 7.9 & 1.4 & & & & & 2.8 & 3 & 2.8 & 0.4 \\
\hline 8125.5 & {$[\mathrm{Cr}$} & $\ldots$ & & 0.8 & 0.4 & 2.0 & 0.7 & 1.6 & 0.1 & 1.3 & 0.2 \\
\hline 8229.8 & {$[\mathrm{Cr} \mathrm{I}$} & & & 3.8 & 1.4 & 3.0 & & 1.3 & 1 & & \\
\hline 8234 & [Fe I & 7.6 & 1.9 & 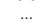 & & 1.3 & 0.5 & 2.0 & & 2.1 & 0.4 \\
\hline & & $\ldots$ & & & & & & & & $\ldots$ & \\
\hline & & 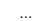 & & 2. & & 33.2 & 14.9 & & & $\ldots$ & \\
\hline & & 24.4 & 1.5 & 7.3 & & 18.0 & 1.0 & 27 & & 18.8 & 4 \\
\hline 8662 & {$[\mathrm{Ca}$} & $\ldots$ & & & & $\ldots$ & & 2.5 & 0.4 & 1.6 & 0.5 \\
\hline 8727.1 & {$[\mathrm{C}$} & $\ldots$ & & 0.4 & 0.2 & $\ldots$ & & 1.2 & 0.1 & 1.2 & 0.1 \\
\hline 8862.8 & H I & & & & & & & 0.3 & 0.2 & & \\
\hline 8891.9 & {$[\mathrm{Fe} \mathrm{II}]$} & 9.8 & 1.0 & 4.1 & 1.4 & 5.5 & 0.7 & 7.5 & 0.4 & 4.9 & 0.8 \\
\hline $\mathrm{F}(\mathrm{H} \beta)$ & $\mathrm{rg} / \mathrm{cm}^{2} /$ & 33.3 & & 353.5 & & 61.8 & & 576.6 & & 54.2 & \\
\hline
\end{tabular}


TABLE A4

Measured line fluXes for N132D Shocked Cloudlets P16 - P20

\begin{tabular}{|c|c|c|c|c|c|c|c|c|c|c|c|}
\hline Cloud ID\#: & & P16 & & P17 & & P18 & & P19 & & P20 & \\
\hline Lambda & ID & Flux & Error & Flux & Error & Flux & Error & Flux & Error & Flux & Error \\
\hline 3736.02 & {$[\mathrm{O} \mathrm{II}]$} & 212.1 & 9.0 & 99.2 & 19.1 & 423.1 & 2.8 & 403.7 & 5.2 & 273.2 & 19.1 \\
\hline 3728.82 & {$\left[\begin{array}{ll}\mathrm{O} & \mathrm{II}\end{array}\right]$} & 120.2 & 10.3 & 411.7 & 22.5 & 91.9 & 2.2 & 105.0 & 3.9 & 146.1 & 13.0 \\
\hline 3835.40 & $\mathrm{H} \eta$ & 63.7 & 10.2 & 10.2 & 1.9 & 9.3 & 0.7 & 3.3 & 2.0 & & \\
\hline 3868.76 & [Ne III] & 48.2 & 2.9 & 21.4 & 2.8 & 39.6 & 0.8 & 40.5 & 1.6 & 82.2 & 8.8 \\
\hline $\begin{array}{l}3888.80 \\
380\end{array}$ & HeI, $\mathrm{H}$ & 58.0 & 6.4 & 17.2 & 3.7 & 22.4 & 0.6 & 18.0 & 1.1 & 33.7 & 12.5 \\
\hline 3933.66 & Ca II & 19.1 & 5.1 & & & 14.9 & 0.4 & 16.3 & 1.9 & & \\
\hline 3969.00 & {$[\mathrm{Ne} \mathrm{III}], \mathrm{Ca} \mathrm{II}, \mathrm{H} \epsilon$} & 79.6 & 6.3 & 40.0 & 5.1 & 37.5 & 0.6 & 28.0 & 1.7 & $\ldots$ & \\
\hline 4026.21 & He I & & & & & 1.0 & 0.2 & 8.8 & 2.0 & & \\
\hline 4068.60 & {$[\mathrm{~S} \mathrm{II}]$} & 40.1 & 1.4 & 32.7 & 1.6 & 38.9 & 0.4 & 36.9 & 1.3 & 9.3 & 4.4 \\
\hline 4076.35 & [S II] & 15.2 & 1.2 & 14.1 & 2.8 & 13.9 & 0.4 & 10.7 & 1.0 & & \\
\hline 4101.74 & $\mathrm{H} \delta$ & 54.9 & 4.3 & 39.4 & 3.0 & 26.8 & 0.5 & 23.5 & 1.2 & 14.3 & 6.4 \\
\hline 4244.00 & {$[\mathrm{Fe} \mathrm{II}]$} & $\ldots$ & & $\ldots$ & & 4.9 & 0.3 & 6.1 & 0.6 & $\ldots$ & \\
\hline 4276.83 & {$[\mathrm{Fe}$ II] } & $\ldots$ & & & & 0.8 & 0.2 & & & & \\
\hline 4287.43 & {$\left[\begin{array}{lll}\mathrm{Fe} & \mathrm{II}\end{array}\right]$} & $\ldots$ & & $\ldots$ & & 4.0 & 0.3 & 4.0 & 0.8 & $\ldots$ & \\
\hline 4319.60 & {$[\mathrm{Fe} \mathrm{II}]$} & & & & & 0.4 & 0.3 & & & & \\
\hline 4340.47 & $\mathrm{H} \gamma$ & 58.2 & 3.2 & 53.1 & 2.2 & 47.4 & 0.8 & 41.5 & 1.8 & 44.8 & 5.1 \\
\hline 4359.14 & {$[\mathrm{Fe}$ IX] } & & & & & 8.0 & 1.5 & 4.8 & 1.4 & & \\
\hline 4363.21 & [O III] & 19.6 & 4.5 & 10.7 & 1.5 & 4.7 & 0.4 & 6.6 & 1.2 & 84.2 & 3.6 \\
\hline 4416.27 & [Fe II] & & & 2.1 & 0.9 & 5.6 & 0.4 & 3.3 & 0.5 & & \\
\hline 4452.38 & [Fe II & ... & & $\ldots$ & & 1.6 & 0 & 1.6 & .7 & ... & \\
\hline 4457.95 & {$[\mathrm{Fe}$ II] } & $\cdots$ & & ... & & 1.1 & 0.2 & 0.9 & 0.3 & ... & \\
\hline 4471.50 & $\mathrm{HeI}$ & 1.3 & 0.5 & & & 5.6 & 0.4 & 4.7 & 0.4 & & \\
\hline 4658.50 & $\mathrm{III}],[\mathrm{Fe} \mathrm{III}]$ & 4.3 & 0.7 & 4.9 & 1.4 & 5.9 & 0.2 & 6.3 & 0.4 & 11.0 & 2.3 \\
\hline 4685.70 & He II & 7.7 & 1.0 & 3.1 & 1.0 & 6.1 & 0.2 & 5.5 & 0.5 & 12.9 & 3.1 \\
\hline 4701.53 & [Fe III] & $\ldots$ & & $\begin{array}{l}.1 \\
\ldots\end{array}$ & & 1.7 & 0.3 & 1.2 & 0.3 & 6.9 & $\begin{array}{l}2.1 \\
2.8\end{array}$ \\
\hline 4754.68 & {$[\mathrm{Fe} \mathrm{III}]$} & 2.4 & 0.9 & & & 1.2 & 0.2 & 1.4 & 0.4 & & \\
\hline 4814.54 & {$[\mathrm{Fe} \mathrm{II}]$} & & & 12.7 & 4.3 & 2.0 & 0.2 & 1.8 & 4 & & \\
\hline 4861.29 & $\mathrm{H} \beta$ & 100.0 & 1.8 & 100.0 & 1.6 & 100.0 & 1.7 & 100.0 & 2.9 & 100.0 & 3.4 \\
\hline 4881.00 & [Fe III] & 2.6 & 0.9 & $\ldots$ & & 2.8 & 0.7 & 1.5 & 0.7 & $\ldots$ & 0.4 \\
\hline 4889.62 & {$[\mathrm{Fe} \mathrm{II}]$} & $\ldots$ & & $\ldots$ & & 2.2 & 0.6 & & & ... & \\
\hline 4905.35 & {$[\mathrm{Fe} \mathrm{II}]$} & & & & & 1.2 & 0.8 & & & & \\
\hline 4958.91 & {$\left[\begin{array}{lll}\mathrm{O} & \mathrm{III}\end{array}\right]$} & 85.8 & 1.2 & 38.8 & 1.4 & 40.4 & 1.3 & 40.3 & 1.0 & 326.2 & 4.0 \\
\hline 5006.84 & {$[\mathrm{O} \quad \mathrm{III}]$} & 246.5 & 2.6 & 134.8 & 2.1 & 123.4 & 2.9 & 130.0 & 1.8 & 967.4 & 6.6 \\
\hline 5111.64 & {$[\mathrm{Fe}$ II] } & & & & & 1.3 & 0.2 & 0.9 & 0.4 & & \\
\hline 5158.79 & {$[\mathrm{Fe} \mathrm{II}],[\mathrm{Fe} \mathrm{VII}]$} & 5.4 & 0.7 & 7.4 & 1.4 & 12.1 & 0.3 & 9.2 & .5 & 11.3 & 2.7 \\
\hline 5198.80 & {$[\mathrm{~N} \mathrm{I}]$} & & & 3.8 & 1.5 & 3.7 & 0.2 & 3.7 & 19 & & \\
\hline 5261.66 & {$[\mathrm{Fe} \mathrm{II}]$} & $\ldots$ & & 2.2 & 0.6 & 4.2 & 0.2 & 3.1 & 0.4 & $\ldots$ & \\
\hline 5270.40 & [Fe III] $]$ & $\ldots$ & & & & 4.4 & 0.6 & 2.3 & 0.8 & & \\
\hline 5273.36 & {$[\mathrm{Fe} \mathrm{II}]$} & & & & & 0.4 & 0.6 & $\ldots$ & & & \\
\hline 5303.06 & {$[\mathrm{Fe}$ XIV] } & 3.2 & 1.3 & $\ldots$ & & 1.8 & 0.2 & & & 4.2 & 1.7 \\
\hline 5334.50 & {$[\mathrm{Fe} \mathrm{II}],[\mathrm{Fe} \mathrm{VI}]$} & 3.9 & 1.3 & & & 2.2 & 0.2 & 2.0 & 0.3 & & \\
\hline 5376.00 & {$[\mathrm{Fe} \mathrm{II}],[\mathrm{Fe}$ VIII] } & 1.1 & 0.5 & & & 0.7 & & 2.8 & 1 & & \\
\hline 5527.30 & & $\ldots$ & & 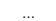 & & 1.9 & & 2 & & & \\
\hline 5754.59 & {$[\mathrm{~N} \mathrm{I}$} & & & 11.4 & 4.0 & 2.2 & 0.1 & 2.5 & 0.5 & 8.0 & 2.2 \\
\hline 5875.61 & He I & 7.0 & 0.7 & 7.9 & 1.7 & 10.9 & 0.2 & 11.5 & 0.5 & 6.0 & 3.5 \\
\hline 6086.97 & {$[\mathrm{Fe}$ VII $]$} & 1.8 & 0.6 & & & 0.8 & 0.1 & & & & \\
\hline 6300.30 & {$\left[\begin{array}{ll}\mathrm{O} & \mathrm{I}\end{array}\right]$} & 78.1 & 2.8 & 97.4 & 3.9 & 96.4 & 0.6 & 74.4 & 1.0 & $\ldots$ & \\
\hline 6312.06 & [S III] & 3.1 & 1.8 & 17.4 & & 2.2 & 0.9 & & & ... & \\
\hline 6363.78 & {$[\mathrm{O} \mathrm{I}]$} & 20.0 & 0.8 & 20.5 & 3.9 & 31.6 & 0.3 & 27.5 & 0.6 & $\ldots$ & \\
\hline 6374.52 & {$[\mathrm{Fe} \mathrm{X}]$} & 3.2 & 0.9 & & & 2.3 & & & & & \\
\hline 6548.05 & {$[\mathrm{~N} \mathrm{II}]$} & 29.3 & 1.2 & 36.1 & 1.4 & 31.6 & 2 & 32.5 & .3 & 33.4 & 2.4 \\
\hline 6562.82 & $\mathrm{H} \alpha$ & 298.0 & 2.1 & 300.9 & 3.3 & 298.9 & 3.0 & 300.7 & .0 & 294.6 & 3.9 \\
\hline 6583.45 & {$[\mathrm{~N} \mathrm{II}]$} & 86.3 & 1.0 & 114.1 & 1.5 & 98.4 & 1.6 & 99.0 & 3.0 & 95.2 & 1.6 \\
\hline 6678.15 & $\mathrm{He} \mathrm{I}$ & 2.8 & 0.5 & 2.5 & 0.4 & 2.9 & 0.1 & 3.4 & 0.3 & & \\
\hline 6716.44 & {$[\mathrm{~S}$} & 61.4 & 0.6 & 113.9 & 1.4 & 87.5 & 1.9 & 92.3 & 2.9 & 121.2 & 2.7 \\
\hline 6730.82 & [S & 109.6 & 0.6 & 165.1 & 2.0 & 132.8 & 2.0 & 145.6 & 4.1 & 141.6 & 3.0 \\
\hline 7065.25 & $\mathrm{HeI}$ & 4.1 & 0.5 & 0.8 & 0.4 & 4.4 & 0.1 & 3.9 & 0.2 & & \\
\hline 7135.79 & {$[\mathrm{Ar} \mathrm{III}]$} & 9.2 & 0.8 & 3.8 & 0. & 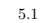 & & & 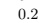 & 16.6 & 1.6 \\
\hline 7155. & & 9.1 & 0. & 2.0 & 0 & 18 & & 17.7 & & 2.6 & 8.8 \\
\hline 7172 & [F & 2.1 & 0 & 0.9 & 0.3 & 3. & 2 & 3.7 & 2 & $\ldots$ & \\
\hline 7291 & {$[\mathrm{Ca}$ II] } & 7.4 & 0.8 & 0.8 & 0.4 & 26.9 & 0.3 & 26.4 & 6 & & \\
\hline 7321.50 & {$\left[\begin{array}{lll}\mathrm{Ca} & \mathrm{II}\end{array}\right],[\mathrm{O} \mathrm{II}]$} & 37.7 & 1.2 & 28.2 & 1.4 & 42.1 & 0.4 & 40.9 & 1.6 & 45.9 & 4.4 \\
\hline 7330.00 & & 27.5 & 1.4 & 19.6 & 1.0 & 19.4 & 0.3 & 12.6 & 0.9 & 24.8 & 2.6 \\
\hline 7377.82 & {$\left[\begin{array}{lll}\mathrm{Ni} & \mathrm{II}\end{array}\right]$} & 3.2 & 0.4 & & & 8.2 & 0.2 & 7.7 & 0.3 & 6.9 & 2.0 \\
\hline 7388.20 & {$[\mathrm{Fe} \mathrm{II}]$} & 1.7 & 0.8 & $\ldots$ & & 2.8 & 0.2 & 2.0 & 0.3 & & \\
\hline 7411. & iNi & & & $\ldots$ & & . & & & & $\ldots$ & \\
\hline 7452 & {$[\mathrm{Fe}$} & 3.1 & 0.6 & & & 5 & & 5.4 & .3 & & \\
\hline 763 & $\mathrm{Fe}$ & 3.0 & 1.6 & & & 2 & 0.1 & 2.3 & .4 & & \\
\hline & & & & $\ldots$ & & 0.6 & 0.1 & & & $\ldots$ & \\
\hline 7686 & & 1.2 & 0.5 & & & 1.7 & 0.1 & 1.6 & 0.2 & & \\
\hline 7891.86 & {$[\mathrm{~F}$} & $\ldots$ & & $\ldots$ & & 1.6 & 0.2 & 3.5 & 1.0 & $\ldots$ & \\
\hline 8125.50 & {$[\mathrm{Cr} \mathrm{I}$} & $\ldots$ & & $\ldots$ & & 1.1 & 0.1 & 1.4 & 0.3 & $\ldots$ & \\
\hline 8229.80 & {$\left[\begin{array}{ll}\mathrm{Cr} & \mathrm{II}\end{array}\right]$} & & & & & 0.6 & 0.1 & & & & \\
\hline 8234.5 & Fe I & & & & & . & & 0.5 & 0.1 & & \\
\hline & & 0.3 & 0.3 & $\ldots$ & & & & $\ldots$ & & $\ldots$ & \\
\hline & & & & & & & & & & & \\
\hline & & 12.9 & 1. & & & 23.4 & & $\ldots$ & & 12.6 & 2.9 \\
\hline $\begin{array}{l}8062.93 \\
8662.14\end{array}$ & {$\left[\begin{array}{lll}\mathrm{Ca} & \mathrm{II}\end{array}\right]$} & 2.1 & 0.8 & $\ldots$ & & $\begin{array}{l}20.4 \\
2.4\end{array}$ & 0.4 & $\cdots$ & & 12.0 & 2.9 \\
\hline 8727.13 & {$\left[\begin{array}{lll}\mathrm{C} & ]\end{array}\right]$} & 2.9 & 0.7 & $\ldots$ & & 1.0 & 0.1 & 1.2 & 0.2 & $\ldots$ & \\
\hline 8862.78 & $\mathrm{HI}$ & & & & & 0.5 & 0.2 & & & & \\
\hline 8891.93 & {$[\mathrm{Fe} \mathrm{II}]$} & 3.4 & 2.2 & $\ldots$ & & 5.4 & 0.6 & 4.9 & 0.4 & 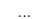 & \\
\hline $\mathrm{F}(\mathrm{H} \beta) \times$ & $\mathrm{rg} / \mathrm{cm}^{2} / \mathrm{s}$ & 53.6 & & 47.7 & & 544.3 & & 116.8 & & 23.5 & \\
\hline
\end{tabular}

